DESY 99-027

ISSN 0418-9833

$\mathrm{DTP} / 99 / 10$

LUNFD6/(NFFL-7166) 1999

\title{
Quark-Antiquark-Gluon Jets in DIS Diffractive Dissociation
}

\author{
J. Bartels ${ }^{a}$ H H. Jung ${ }^{b}$ and M. Wüsthoff ${ }^{1}$ \\ ${ }^{a}$ II. Inst. f. Theoretische Physik, Univ. Hamburg, Luruper Chaussee 149, D-22761 Hamburg \\ ${ }^{b}$ Department of Physics, Lund University, 22100 Lund, Sweden \\ ${ }^{c}$ Department of Physics, University of Durham, Durham DH1 3LE, UK
}

\begin{abstract}
We study the diffractive production of $q \bar{q} g$ jets with large transverse momenta in the region of large diffractive masses (small $\beta$ ). Cross sections for transverse and longitudinal photons are obtained in the leading $\log 1 / x_{\mathbb{P}}$ and $\log 1 / \beta$ approximation, keeping all powers in $\log k_{t}^{2} / Q^{2}$. We perform a numerical study and illustrate the angular distribution of the three jets. We also estimate the integrated diffractive three jet cross section and compare with the dijet cross section obtained before.
\end{abstract}

\section{Introduction}

The diffractive dissociation in deep inelastic scattering lies on the interface between perturbative and non-perturbative QCD, and one expects the diffractive cross section to contain both hard and soft contributions. As a first experimental hint in this direction, one might take the observation that the energy dependence of the diffractive cross section, i.e. the measured intercept of the Pomeron flux factor tends to be larger than that of the pure soft Pomeron [1, 2]. As a possible interpretation of this observation, the contribution of the "perturbative Pomeron", the gluon structure function, may turn out to be substantial: the observed rise of the gluon structure function at small - $x$ enhances the hard part of the diffractive final state and thus leads to an effective Pomeron intercept which is bigger than that of the soft Pomeron.

In a first attempt to provide a description of the diffractive cross section which takes into account both hard and soft contributions a parameterization has been suggested [3] and successfully fitted to both ZEUS and H1 [3, [4] data. As a main result, it was shown that an acceptable description of the data requires (at least) three pieces: the diffractive production of transverse $q \bar{q}$ and $q \bar{q} g$ states and the diffractive production of longitudinal vector mesons. The fits also confirmed that the effective intercept of the Pomeron tends to be larger than the soft Pomeron.

In order to obtain further insight into DIS diffraction and, in particular, to understand the origin of the higher Pomeron intercept, one has to open the diffractive final state and to isolate those parts which can be attributed to the hard Pomeron. Apart from the longitudinal vector particles which

\footnotetext{
${ }^{1}$ Supported by the TMR Network "QCD and Deep Structure of Elementary Particles"
} 
have been suggested already several years ago [5], diffractive final states consisting of hard jets only, are the most promising candidates. As a first step, the production of quark-antiquark jets (Fig. 11) with large transverse momenta has been discussed by several groups [6, 0, 8]. To summarize the main result, the energy dependence is given by the square of the gluon structure function:

$$
d \sigma \sim\left[\frac{1}{k_{T}^{2}} x_{\mathbb{P}} g\left(x_{\mathbb{P}}, k_{T}^{2} /(1-\beta)\right)\right]^{2}
$$

where $k_{T}$ denotes the transverse momentum of the quarks in the photon - Pomeron rest frame, and $\beta=Q^{2} /\left(Q^{2}+M^{2}\right)$ is related to the mass $M$ of the diffractive system. From eq. (1.1) one concludes that for small masses ( $\beta$ near 1) the cross section will be dominated by this "hard Pomeron ", either in the production of vector particles or of hard $q \bar{q}$-jets. For larger diffractive masses the contribution of hard $q \bar{q}$ jets is smaller but not negligible. Another interesting feature is the azimuthal dependence of the $q \bar{q}$ cross section. It was found [9] that, in the photon - Pomeron rest frame, the two jets prefer to lie perpendicular to the scattering plane (defined by the photon - Pomeron direction and the outgoing electron momentum). This has to be compared with the photon - gluon fusion mechanism in the usual DIS final state, where the two outgoing quarks prefer to lie inside the scattering plane. The experimental verification of these theoretical predictions turns out to be rather difficult: to make the calculation applicable, the jet final state is not allowed to contain any soft Pomeron remnants; the presence of such pieces changes the situation rather radically. To exclude such a soft remnant requires high statistics of the data and a rather good resolution in the jet algorithm. First results have been reported in [10].

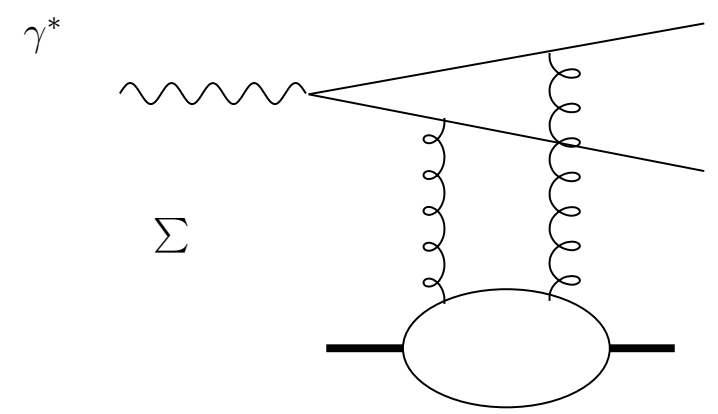

Figure 1: Dijet Production. The exchanged gluons couple to the quarks in all possible ways.

For not so small diffractive masses ( $\beta$ away from 1 ) one expects the production of extra gluons to become essential. The simplest final state consists of the $q \bar{q}$ pair and one gluon; in the region of large diffractive masses ( $\operatorname{small} \beta$ ) one expects a separation in rapidity between the $q \bar{q}$ system and the gluon (Fig. 2) (not to be confused with the rapidity gap between the diffractive $q \bar{q} g$ system and the outgoing proton). In order to isolate the "hard Pomeron" contributions one, again, wants to study final states which consist only of jets with large transverse momenta (and no further soft remnants). In this paper we present the calculation of this three-jet cross section, restricting ourselves to the region of large diffractive masses (small $\beta)$. We shall work in the leading $-\log (1 / \beta), \log \left(1 / x_{\mathbb{P}}\right)$ approximation, retaining all powers in $k_{T}^{2} / Q^{2}$. This approximation includes also higher-twist terms of the diffractive cross section. Diffractive production of $q \bar{q} g$ final states in another kinematic region has been studied before [11, 12]: the gluon transverse momentum has to be smaller than those of the quarks. The results of [12] extend into the large $\beta$-region. 


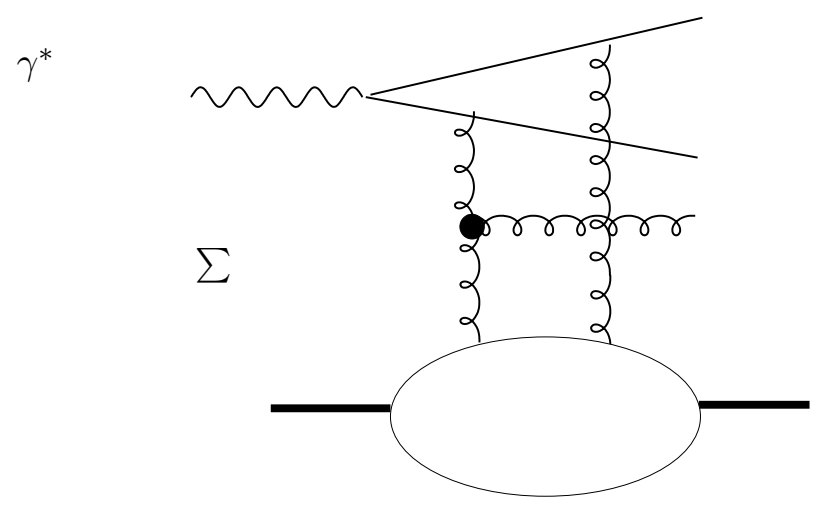

Figure 2: Three jet Production. The exchanged gluons couple to the quarks in all possible ways.

There are several questions to which our jet analysis gives an answer. Apart from a quantitative estimate of the jet rates (as a function of the imposed kinematical cuts) our analytic formula allows a first step towards an analysis of the event shape of diffractive final states (e.g. thrust). In particular, we find that in the region of medium small $\beta$ (where our formula is applicable) the $q \bar{q} g$ final state provides a substantial contribution to the two-jet cross section. Our framework also allows to address the transition from hard jets to the aligned gluon configuration: in a future step one might model a two gluon amplitude which allows to extrapolate our formula into the soft Pomeron region. Such a model (an explicit parameterization was suggested in ref. [13]) would allow to describe both the hard and the soft region of diffractive $q \bar{q} g$ production. However, a severe limitation is our restriction to the small- $\beta$ region. In order to cover the full $\beta$ range we need a complete LO calculation of three parton final states in DIS diffraction which includes a NLO two jet calculation.

This paper will be organized as follows. In section 2 we describe our calculation and present the result for the three jet production cross section. Section 3 contains the transformation into the impact parameter space and in section 4 we discuss a few particular kinematic regions for which our formula allows analytic approximations. In section 5 we perform a first numerical analysis, calculate integrated cross sections and show a few plots. In the final section we summarize our results and make a few concluding remarks.

\section{The Differential Jet Production Cross Section}

Our notations are illustrated in Fig. 3. As usual, $q$ and $p$ denote the four momenta of the photon and the proton, respectively, $t=r^{2}$ is the square of the momentum transferred from the proton to the diffractive system. The total energy is denoted by $s$ and the sub-energy of the $\gamma^{*}$-proton system with $W^{2}$. The scaling variables are $x=Q^{2} / 2 p q, x_{\mathbb{P}}=\left(Q^{2}+M^{2}\right) /\left(Q^{2}+W^{2}\right)$, and $\beta=Q^{2} /\left(Q^{2}+M^{2}\right)$. For simplicity we restrict ourselves to the forward direction, $t=0$, such that the four momentum $r=x_{\mathbb{P}} p$ has no transverse component. We use the Sudakov parameterization

$$
k_{i}=\alpha_{i} q^{\prime}+\beta_{i} p+k_{i T}, \mathbf{k}_{i}^{2}=-k_{i T}^{2},
$$

where $q^{\prime}=q+x p$. We work in a reference frame where photon and proton momenta are collinear (along the $z$-direction); the scattering plane (the $x-z$ plane) is defined by the incoming and 


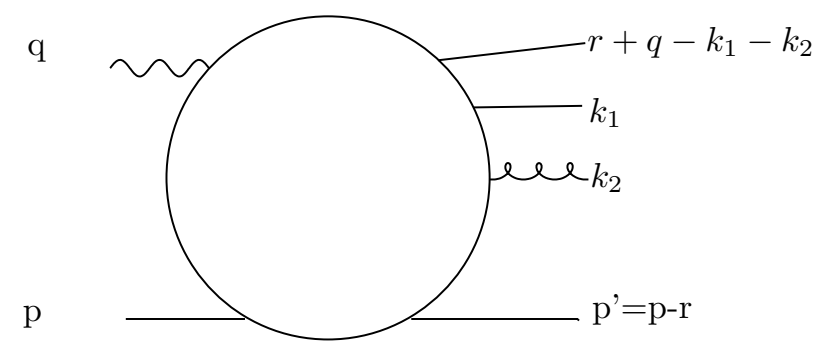

Figure 3: Notations for the diffractive production of a $q \bar{q} g$ system.

outgoing electron. Azimuthal angles refer to this scattering plane. In order to justify the use of perturbation theory we start in the "hard region": all three final state partons have large transverse momenta. Later on we will show that the more precise conditions imply that the virtualities of the exchanged quark and gluon have to be large. This leads to:

$$
\begin{aligned}
Q_{0}^{2} & <\mathbf{k}_{2}^{2} \\
Q_{0}^{2} & <\frac{1}{\alpha_{1}}\left[\alpha_{1}\left(1-\alpha_{1}\right) Q^{2}+\mathbf{k}_{1}^{2}\right] \\
Q_{0}^{2} & <\frac{1}{1-\alpha_{1}}\left[\alpha_{1}\left(1-\alpha_{1}\right) Q^{2}+\left(\mathbf{k}_{1}+\mathbf{k}_{2}\right)^{2}\right]
\end{aligned}
$$

Our calculation will be done in the leading - $\log W^{2}$, leading - $\log M^{2}$ approximation and applies to the region $Q^{2} \ll M^{2} \ll W^{2}$. As usual, in this approximation we have $\alpha_{2} \ll \alpha_{1}, 1-\alpha_{1}$ and $\beta_{2}$ close to $x_{\mathbb{P}}$. For large values of $\log Q^{2} / \mathbf{k}_{i}^{2}$ our approximation reduces to the double logarithmic approximation (DLA). The invariant mass $M$ of the diffractive system can be expressed (in this leading log approximation) as

$$
M^{2}=W^{2}\left(x_{\mathbb{P}}-x\right)
$$

The mass of the $q \bar{q}$ subsystem is given by $m_{q \bar{q}}^{2}=m^{2}-\mathbf{k}_{2}^{2}$ with

$$
m^{2}=M^{2}-\beta_{2} W^{2}, \quad \beta_{2}=\frac{M^{2}-m^{2}}{W^{2}} .
$$

For $Q^{2} \ll M^{2}$ (i.e. small $\beta$ ) we expect the invariant mass of the $q \bar{q}$ pair to be much smaller than $M^{2}$ (of the order of $Q^{2}$ ). From the mass shell condition of the upper quark line (with momentum $\left.r+q-k_{1}-k_{2}\right)$ we have the relation:

$$
\begin{aligned}
0 & =\alpha_{1}\left(1-\alpha_{1}-\alpha_{2}\right)\left(M^{2}-\beta_{2} W^{2}\right)-\left(1-\alpha_{1}-\alpha_{2}\right) \mathbf{k}_{1}^{2}-\alpha_{1}\left(\mathbf{k}_{1}+\mathbf{k}_{2}\right)^{2} \\
& \approx \alpha_{1}\left(1-\alpha_{1}\right) m^{2}-\left(1-\alpha_{1}\right) \mathbf{k}_{1}^{2}-\alpha_{1}\left(\mathbf{k}_{1}+\mathbf{k}_{2}\right)^{2}
\end{aligned}
$$

We begin with the calculation of the $\gamma^{*} p$ cross section. The diagrams we have to calculate are shown in Fig. 4 . Since we consider the square of the scattering matrix element and, moreover, take the discontinuity in $W^{2}$, all quark lines between the leftmost and the rightmost gluon are on-shell; the same holds for the produced gluon below the quark loop. This leads to $\delta$-functions which will be used to integrate over all $\alpha_{i}$ and $\beta_{i}$, except for $\beta_{2}$. We begin with the transverse cross section. Let $e_{x}=(0,1,0,0)=\left(0, \mathbf{e}_{x}, 0\right)$ and $e_{y}=(0,0,1,0)=\left(0, \mathbf{e}_{y}, 0\right)$ be the transverse polarization vectors of 
the photon (it should be noted that $e_{x}$ lies in the scattering plane). Contracting the Lorentz indices of the two external photons with the transverse polarization vectors $e_{1}$ and $e_{2}$ (each of them can be either $e_{x}$ or $e_{y}$ ), the sum of the diagrams in Fig. $⿴$ leads to a contribution of the form

$$
2 W^{4} \delta\left(\left(r+q-k_{1}-k_{2}\right)^{2}\right)\left[4 \alpha_{1}^{2}\left(1-\alpha_{1}\right)^{2} \mathbf{e}_{1 i} \mathbf{e}_{2 j} M_{i l} M_{j l}^{\prime}-\alpha_{1}\left(1-\alpha_{1}\right) \mathbf{e}_{1} \cdot \mathbf{e}_{2} M_{i l} M_{i l}^{\prime}\right]
$$

(here $i, j, l=1,2$ refer to the transverse components; repeated indices are summed over, and the prime at the second $M_{i l}^{\prime}$ factor indicates that it belongs to the two-gluon amplitude with momentum $\mathbf{l}^{\prime}$, attached to the lower two gluons on the rhs of the diagrams in Fig.4). We have exhibited the $\delta$ function of the upper quark line which leads to the condition eq. (2.4) and will be used to express $\alpha_{1}$ in terms of $M, \beta_{2}, \mathbf{k}_{1}$, and $\mathbf{k}_{2}$. The expression for $M_{i j}$ will be discussed below. Contracting this with the lepton tensor $\frac{y^{2}}{4 Q^{2}} L_{\mu \nu} e_{1}^{\mu} e_{2}^{\nu}$ and summing over the two possible transverse polarizations we obtain

$$
\begin{gathered}
2 W^{4} \delta\left(\left(r+q-k_{1}-k_{2}\right)^{2}\right) \\
\cdot\left[\frac{1+(1-y)^{2}}{2} 4 \alpha_{1}\left(1-\alpha_{1}\right)\left[\alpha_{1}^{2}+\left(1-\alpha_{1}\right)^{2}\right] M_{i l} M_{i l}^{\prime}-4(1-y) \alpha_{1}^{2}\left(1-\alpha_{1}\right)^{2}\left(M_{1 l} M_{1 l}^{\prime}-M_{2 l} M_{2 l}^{\prime}\right)\right]
\end{gathered}
$$

In the second line one recognizes two terms. The first one (proportional to $M_{i l} M_{i l}^{\prime}$ ) corresponds to the sum over the two transverse polarizations, the second one (proportional to $M_{1 l} M_{1 l}^{\prime}-M_{2 l} M_{2 l}^{\prime}$, $M_{1 l}=\mathbf{e}_{x i} M_{i l}, M_{2 l}=\mathbf{e}_{y i} M_{i l}$ ) to the difference. As long as we do not integrate over the azimuthal angle of the outgoing electron, the two transverse polarizations of the photon come with different weights, and that is why we write our result in terms of both the sum and the difference of the two polarizations.

For the longitudinal cross section we arrive at the following structure:

$$
2 W^{4} \delta\left(\left(r+q-k_{1}-k_{2}\right)^{2}\right) \alpha_{1}^{3}\left(1-\alpha_{1}\right)^{3} Q^{2} M_{l} M_{l}^{\prime} .
$$

Together with the lepton tensor we obtain

$$
2 W^{4} \delta\left(\left(r+q-k_{1}-k_{2}\right)^{2}\right) 2(1-y) \alpha_{1}^{3}\left(1-\alpha_{1}\right)^{3} Q^{2} M_{l} M_{l}^{\prime} .
$$

Finally, we have the interference between the transverse and the longitudinal photon. The expressions analogous to eq. (2.5) and eq. (2.6) are given by

$$
2 W^{4} \delta\left(\left(r+q-k_{1}-k_{2}\right)^{2}\right) 2 \sqrt{Q^{2}} \alpha_{1}^{2}\left(1-\alpha_{1}\right)^{2}\left(1-2 \alpha_{1}\right) M_{1 l} M_{l}^{\prime} .
$$

and

$$
2 W^{4} \delta\left(\left(r+q-k_{1}-k_{2}\right)^{2}\right) \sqrt{1-y}(2-y) \sqrt{Q^{2}} \alpha_{1}^{2}\left(1-\alpha_{1}\right)^{2}\left(1-2 \alpha_{1}\right) M_{1 l} M_{l}^{\prime} .
$$

Before we turn to the calculation of the $M_{i l}$ and $M_{l}$, we write down the final formula for the process $e+p \rightarrow e^{\prime}+q \bar{q} g+p$. The diffractive final state is characterized by the invariant mass $M$, the longitudinal momentum fraction $\beta_{2}$ of the outgoing gluon, and by the transverse momenta $\mathbf{k}_{1}$, $\mathbf{k}_{2}$ of the outgoing quark and gluon, respectively (in our notation, $\mathbf{k}^{2}=-k_{T}^{2}>0$ ). Starting from 


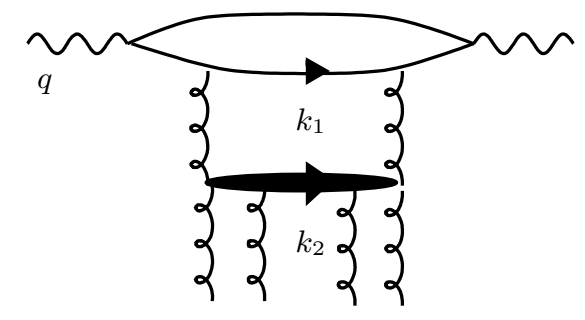

$$
l+r l \quad l^{\prime} \quad l^{\prime}+r
$$

a
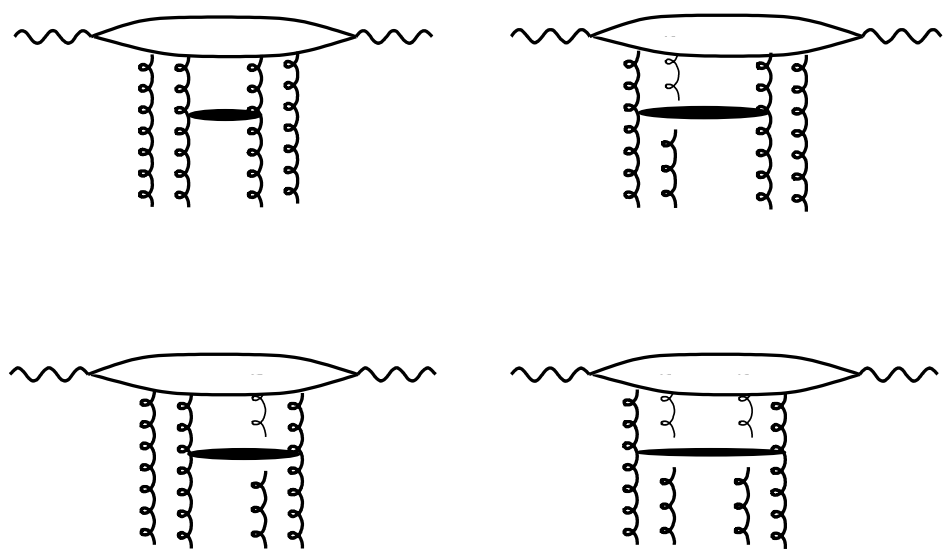

$\mathrm{b}$
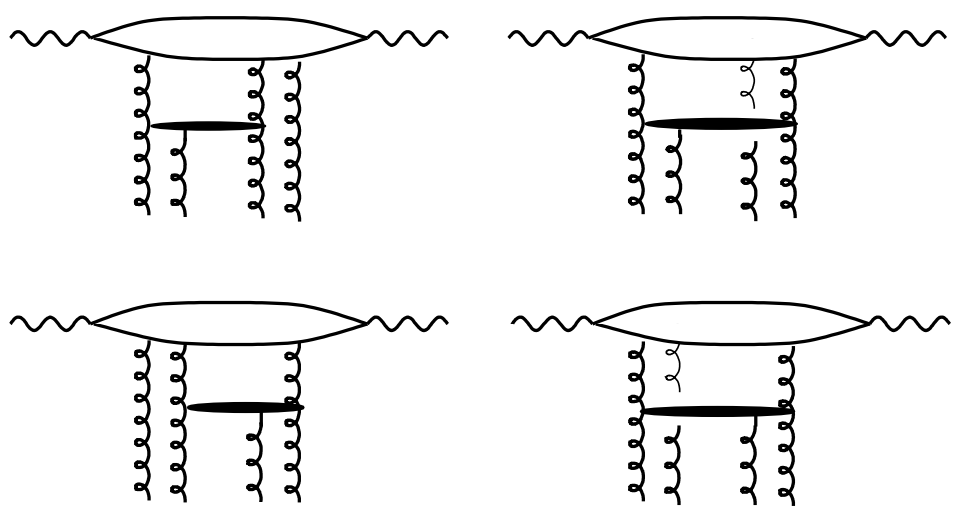

Figure 4: Diagrams for the cross section of diffractive $q \bar{q} g$ production. We only show the diffractive system: at the lower end the two-gluon systems on both sides couple to the gluon structure function of the proton. At the upper end, the gluons couple to the quark loop in all possible ways. We do not show the vertical discontinuity line which divides each diagram into left and right hand pieces. 
eq. (2.5) - eq. (2.10) and evaluating the final state phase space integrals we obtain:

$$
\begin{aligned}
& \frac{d \sigma_{D}^{e^{-} p}}{d y d Q^{2} d M^{2} d m^{2} d^{2} \mathbf{k}_{1} d^{2} \mathbf{k}_{2} d t}{ }_{\mid t=0}=\frac{\alpha_{e m}}{y Q^{2} \pi} \\
& \cdot\left[\frac{1+(1-y)^{2}}{2} \frac{d \sigma_{D, T,+}^{\gamma^{*} p}}{d M^{2} d m^{2} d^{2} \mathbf{k}_{1} d^{2} \mathbf{k}_{2} d t}{ }_{\mid t=0}-2(1-y) \frac{d \sigma_{D, T,-}^{\gamma^{*} p}}{d M^{2} d m^{2} d^{2} \mathbf{k}_{1} d^{2} \mathbf{k}_{2} d t}{ }_{\mid t=0}\right. \\
& \left.+(1-y){\frac{d \sigma_{D, L}^{\gamma^{*} p}}{d M^{2} d m^{2} d \mathbf{k}_{1} d^{2} \mathbf{k}_{2} d t}}_{\mid t=0}+(2-y) \sqrt{1-y} \frac{d \sigma_{D, I}^{\gamma^{*} p}}{d M^{2} d m^{2} d^{2} \mathbf{k}_{1} d^{2} \mathbf{k}_{2} d t}{ }_{\mid t=0}\right],
\end{aligned}
$$

where the $\gamma^{*} p$ cross sections are defined as:

$$
\begin{aligned}
\left.\frac{d \sigma_{D, T+}^{\gamma^{*} p}}{d M^{2} d m^{2} d^{2} \mathbf{k}_{1} d^{2} \mathbf{k}_{2} d t}\right|_{t=0} & =\frac{9}{128 \pi} \frac{1}{\sqrt{S}\left(M^{2}-m^{2}\right) m^{2}} \sum_{f} e_{f}^{2} \alpha_{e m} \alpha_{s}^{3}\left[\alpha_{1}^{2}+\left(1-\alpha_{1}\right)^{2}\right] \alpha_{1}\left(1-\alpha_{1}\right) \\
\frac{d \sigma_{D, T-}^{\gamma^{*} p}}{d M^{2} d m^{2} d^{2} \mathbf{k}_{1} d^{2} \mathbf{k}_{2} d t} & =\frac{9}{128 \pi} \frac{1}{\sqrt{S}\left(M^{2}-m^{2}\right) m^{2}} \sum_{f} e_{f}^{2} \alpha_{e m} \alpha_{s}^{3} \alpha_{1}^{2}\left(1-\alpha_{1}\right)^{2} \\
\frac{d \sigma_{D, L}^{\gamma^{*} p}}{d M^{2} d m^{2} d^{2} \mathbf{k}_{1} d^{2} \mathbf{k}_{2} d t} & =\frac{9}{128 \pi} \frac{\left(M_{1 l} M_{1 l}^{\prime}-M_{2 l} M_{2 l}^{\prime}\right)}{\sqrt{S}\left(M^{2}-m^{2}\right) m^{2}} \sum_{f} e_{f}^{2} \alpha_{e m} \alpha_{s}^{3} 4 \alpha_{1}^{3}\left(1-\alpha_{1}\right)^{3} Q^{2} M_{l} M_{l}^{\prime} \\
\frac{d \sigma_{D, I}^{\gamma^{*} p}}{d M^{2} d m^{2} d^{2} \mathbf{k}_{1} d^{2} \mathbf{k}_{2} d t} & =\frac{9}{128 \pi} \frac{1}{\sqrt{S}\left(M^{2}-m^{2}\right) m^{2}} \sum_{f} e_{f}^{2} \alpha_{e m} \alpha_{s}^{3} \alpha_{1}^{2}\left(1-\alpha_{1}\right)^{2}\left(1-2 \alpha_{1}\right) \\
& \frac{1}{2} \sqrt{Q^{2}}\left[M_{1 l} M_{l}^{\prime}+M_{l} M_{1 l}^{\prime}\right]
\end{aligned}
$$

Here

$$
S=\left(1+\frac{\mathbf{k}_{1}^{2}}{m^{2}}-\frac{\left(\mathbf{k}_{1}+\mathbf{k}_{2}\right)^{2}}{m^{2}}\right)^{2}-4 \frac{\mathbf{k}_{1}^{2}}{m^{2}},
$$

and the prefactors containing $\alpha_{1}$ have to be re-expressed using the $\delta$-function condition eq. (2.4). As we have discussed before, the transverse cross section has two terms. The first one corresponds to the sum over the two transverse polarizations, the second one to the difference. This second term of the transverse cross section, as well as the interference cross section, introduce the azimuthal dependence of our cross section.

It is instructive to consider the kinematics of the three partons in the final state. Within our approximation, we have to restrict ourselves to the forward direction of the Pomeron: in the $\gamma^{*}$ $\mathbb{P}$ CM system the gluon is emitted in the direction of the Pomeron. The kinematic boundaries of the transverse momenta $\mathbf{k}_{1}$ and $\mathbf{k}_{2}$ are determined by the condition $S>0$ (eq. (2.17)). The allowed region is illustrated in Fig. 5, where we have plotted the two quark transverse momenta, normalized to the invariant mass square $m^{2}$ of the $q \bar{q}$ subsystem: $\mathbf{k}_{1}^{2} / m^{2}$ and $\left(\mathbf{k}_{1}+\mathbf{k}_{2}\right)^{2} / m^{2}$. The curve $S=0$ shows that the quark momenta are essentially restricted by $m^{2}$. For the particular case of small gluon transverse momenta $\mathbf{k}_{2}^{2} \ll \mathbf{k}_{1}^{2}$, we are on the diagonal line $\mathbf{k}_{1}^{2} \approx\left(\mathbf{k}_{1}+\mathbf{k}_{2}\right)^{2}$, and the 


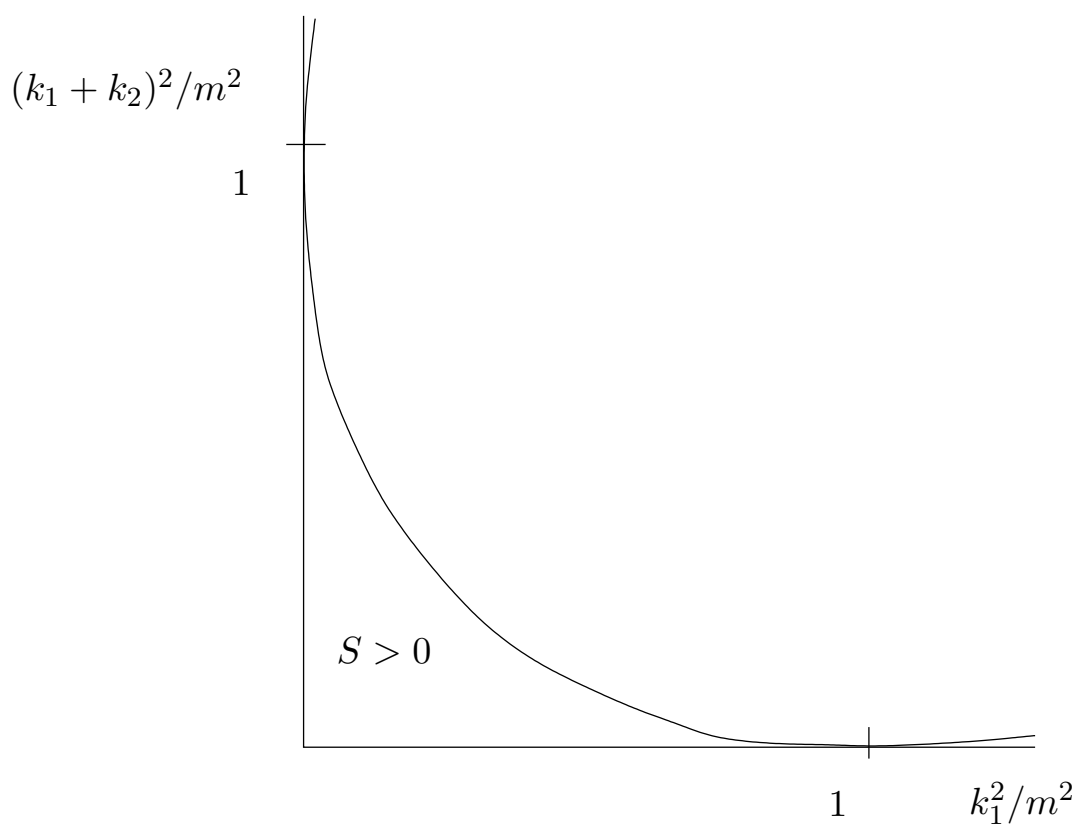

Figure 5: Kinematic Boundaries. The drawn line belongs to $S=0$.

physical region is restricted to $\mathbf{k}_{1}^{2} \leq \frac{1}{4} m^{2}$. For a given set of transverse momenta, the longitudinal momentum fraction $\alpha_{1}$ is given by one of the two values:

$$
\alpha_{1}=\frac{1}{2}\left(1+\frac{\mathbf{k}_{1}^{2}-\left(\mathbf{k}_{1}+\mathbf{k}_{2}\right)^{2}}{m^{2}} \pm \sqrt{S}\right)
$$

$\left(\alpha_{2}, \beta_{1}\right.$, and $\beta_{2}$ follow from the mass shell conditions $\left.k_{1}^{2}=0, k_{2}^{2}=0\right)$. The limiting values $\alpha_{1}=0$ and $1-\alpha_{1}=0$ lie on the axis $\mathbf{k}_{1}^{2}=0$ and $\left(\mathbf{k}_{1}+\mathbf{k}_{2}\right)^{2}=0$, respectively, i.e. they correspond to final states where one of the quark lines becomes soft. For small $\mathbf{k}_{2}^{2}$ (i.e. on the line $\mathbf{k}_{1}^{2}=\left(\mathbf{k}_{1}+\mathbf{k}_{2}\right)^{2}$ ) eq. (2.17) simplifies to

$$
\alpha_{1}=\frac{1}{2}\left(1 \pm \sqrt{1-4 \frac{\mathbf{k}_{1}^{2}}{m^{2}}}\right)
$$

and

$$
\alpha_{1}\left(1-\alpha_{1}\right)=\mathbf{k}_{1}^{2} / m^{2}
$$

For the angle $\Delta$ between the two quarks we find

$$
\frac{1-\cos \Delta}{2}=\frac{\left(\mathbf{k}_{1}+\alpha_{1} \mathbf{k}_{2}\right)^{2} / M^{2}}{\left[\alpha_{1}^{2}+\mathbf{k}_{1}^{2} / M^{2}\right]\left[\left(1-\alpha_{1}\right)^{2}+\left(\mathbf{k}_{1}+\mathbf{k}_{2}\right)^{2} / M^{2}\right]}
$$

In the denominator, the $\alpha_{1}$ terms will dominate, as long as we stay away from the values $\alpha_{1}=0,1$, the numerator is (at most) of the order $m^{2} / M^{2}$ and the opening angle $\Delta=O\left(\mathrm{~m}^{2} / M^{2}\right)$. Therefore, for small $m^{2} / M^{2}$ the opening angle will be small (of the order $\Delta=O\left(m^{2} / M^{2}\right)$ ), and we expect, in the $\gamma^{*}-\mathbb{P}$ CM system, the gluon and the $q \bar{q}$-pair to come out as two back-to-back jets. The $q \bar{q}$ - pair 
produces a broader jet, where the opening angle is given by eq. (2.21) and will approximately be of the order of $O\left(\mathrm{~m}^{2} / \mathrm{M}^{2}\right)$. When $\mathrm{m}^{2}$ gets larger, the $q \bar{q}$ - pair jet becomes broader and eventually can be resolved into two separate jets. The angle of the axis of the two-jet configuration is given by the direction of the outgoing gluon. If $\theta_{2}$ denotes the angle between the gluon and the proton momentum, we find:

$$
\frac{1-\cos \theta_{2}}{2}=\frac{\mathbf{k}_{2}^{2} / M^{2}}{\mathbf{k}_{2}^{2} / M^{2}+\left(1-m^{2} / M^{2}\right)^{2}}
$$

For $m^{2} \ll M^{2}$ the gluon transverse momentum $\mathbf{k}_{2}^{2}$ will be much smaller than $M^{2}$, the angle will be small, i.e. the outgoing gluon moves in the direction of the proton with an angle of the order $O\left(\mathbf{k}_{2}^{2} / M^{2}\right)$. In the strong ordering region $\mathbf{k}_{2}^{2} \ll \mathbf{k}_{1}^{2}$, eq. (2.21) can be approximated by

$$
\frac{1-\cos \Delta}{2}=\frac{m^{2}}{M^{2}} \frac{1}{\left[\alpha_{1}+\left(1-\alpha_{1}\right) m^{2} / M^{2}\right]\left[\left(1-\alpha_{1}\right)+\alpha_{1} m^{2} / M^{2}\right]}
$$

In section 5 we present results of a numerical study of our cross section formula which illustrate the geometry of the three-jet configuration.

Let us now turn to the calculation of the $M_{i l}$ and $M_{l}$. Each of them is of the form

$$
M_{i l}=\int \frac{d^{2} \mathbf{l}}{\pi \mathbf{l}^{2}} \mathcal{F}\left(x_{\mathbb{P}}, \mathbf{l}^{2}\right) T_{i l},
$$

(and an analogous expression for $M_{l}$ ), where $\mathcal{F}$ denotes the unintegrated gluon structure function

$$
\int_{Q_{0}^{2}}^{Q^{2}} d \mathbf{l}^{2} \mathcal{F}\left(x, \mathbf{l}^{2}\right)=x g\left(x, Q^{2}\right)
$$

and models the perturbative Pomeron. In the fermion loop at the top of the diagrams in Fig. 目 it is understood that the gluons couple to the quarks in all possible ways. Since we consider the square of the scattering matrix element and take the discontinuity in $W^{2}$, all quark lines between the leftmost and the rightmost gluon are on - shell; this leads to $\delta$ - functions which will be used to integrate over all $\alpha_{i}$ and $\beta_{i}$, except for $\beta_{2}$. We begin with the transverse cross section. The diagrams with two gluons lines attached to the fermion loop (Fig. $6 \mathrm{a}$ ) lead to:

$$
T_{i l}^{(a)}=2\left(\frac{\mathbf{k}_{1}+\mathbf{k}_{2}}{D\left(\mathbf{k}_{1}+\mathbf{k}_{2}\right)}-\frac{\mathbf{k}_{1}}{D\left(\mathbf{k}_{1}\right)}\right)_{i}\left(\frac{\mathbf{k}_{2}+\mathbf{l}}{\left(\mathbf{k}_{2}+\mathbf{l}\right)^{2}}-\frac{\mathbf{k}_{2}}{\mathbf{k}_{2}^{2}}\right)_{l},
$$

with

$$
D(\mathbf{k})=\alpha_{1}\left(1-\alpha_{1}\right) Q^{2}+\mathbf{k}^{2} .
$$

In eq. (2.26) the first factor (with subscript ${ }^{\prime} i$ ) comes from the fermion loop, the second factor from gluon production below the fermion loop. For the latter we have made use of the $K_{2 \rightarrow 4}$ gluon vertex [16, 17]:

$$
\left(\frac{\mathbf{k}_{2}+\mathbf{l}}{\left(\mathbf{k}_{2}+\mathbf{l}\right)^{2}}-\frac{\mathbf{k}_{2}}{\mathbf{k}_{2}^{2}}\right)_{l}\left(\frac{\mathbf{k}_{2}+\mathbf{l}^{\prime}}{\left(\mathbf{k}_{2}+\mathbf{l}^{\prime}\right)^{2}}-\frac{\mathbf{k}_{2}}{\mathbf{k}_{2}^{2}}\right)_{l}
$$

(this includes the two vertical gluon propagators with momenta $\mathbf{k}_{2}$ ). A similar expression holds for $M_{j l}^{\prime}$. 
Next we turn to the four diagrams in Fig. $4 b$ where four gluons are attached to the fermion loop. We write :

$$
\left(M_{i l} M_{j l}^{\prime}\right)^{(b 1)}=\int \frac{d^{2} \mathbf{l}}{\pi \mathbf{l}^{2}} \mathcal{F}\left(x_{\mathbb{P}}, \mathbf{l}^{2}\right) \int \frac{d^{2} \mathbf{l}^{\prime}}{\pi \mathbf{l}^{\prime 2}} \mathcal{F}\left(x_{\mathbb{P}}, \mathbf{l}^{\prime 2}\right)\left(T_{i l} T_{j l}^{\prime}\right)^{(b 1)}
$$

The first diagram in Fig. $4 b$ leads to:

$$
\begin{aligned}
\left(T_{i l} T_{j l}^{\prime}\right)^{(b 1)} & =\left(\frac{\mathbf{l}+\mathbf{k}_{1}+\mathbf{k}_{2}}{D\left(\mathbf{l}+\mathbf{k}_{1}+\mathbf{k}_{2}\right)}+\frac{\mathbf{l}-\mathbf{k}_{1}}{D\left(\mathbf{l}-\mathbf{k}_{1}\right)}-\frac{\mathbf{k}_{1}+\mathbf{k}_{2}}{D\left(\mathbf{k}_{1}+\mathbf{k}_{2}\right)}+\frac{\mathbf{k}_{1}}{D\left(\mathbf{k}_{1}\right)}\right)_{i}\left(\frac{\mathbf{k}_{2}+\mathbf{l}}{\left(\mathbf{k}_{2}+\mathbf{l}\right)^{2}}-\frac{\mathbf{k}_{2}}{\mathbf{k}_{2}^{2}}\right)_{l} \\
& \cdot\left(\frac{\mathbf{l}^{\prime}+\mathbf{k}_{1}+\mathbf{k}_{2}}{D\left(\mathbf{l}^{\prime}+\mathbf{k}_{1}+\mathbf{k}_{2}\right)}+\frac{\mathbf{l}^{\prime}-\mathbf{k}_{1}}{D\left(\mathbf{l}^{\prime}-\mathbf{k}_{1}\right)}-\frac{\mathbf{k}_{1}+\mathbf{k}_{2}}{D\left(\mathbf{k}_{1}+\mathbf{k}_{2}\right)}+\frac{\mathbf{k}_{1}}{D\left(\mathbf{k}_{1}\right)}\right)_{j}\left(\frac{\mathbf{k}_{2}+\mathbf{l}^{\prime}}{\left(\mathbf{k}_{2}+\mathbf{l}^{\prime}\right)^{2}}-\frac{\mathbf{k}_{2}}{\mathbf{k}_{2}^{2}}\right)_{l}
\end{aligned}
$$

Similarly, we find for the other three contributions:

$$
\begin{aligned}
\left(T_{i l} T_{j l}^{\prime}\right)^{(b 2)}= & -\left(\frac{\mathbf{l}-\mathbf{k}_{1}-\mathbf{k}_{2}}{D\left(\mathbf{l}-\mathbf{k}_{1}-\mathbf{k}_{2}\right)}+\frac{\mathbf{l}+\mathbf{k}_{1}}{D\left(\mathbf{l}+\mathbf{k}_{1}\right)}-\frac{\mathbf{k}_{1}+\mathbf{k}_{2}}{D\left(\mathbf{k}_{1}+\mathbf{k}_{2}\right)}+\frac{\mathbf{k}_{1}}{D\left(\mathbf{k}_{1}\right)}\right)_{i}\left(\frac{\mathbf{k}_{2}-\mathbf{l}}{\left(\mathbf{k}_{2}-\mathbf{l}\right)^{2}}-\frac{\mathbf{k}_{2}}{\mathbf{k}_{2}^{2}}\right)_{l} \\
& \cdot\left(\frac{\mathbf{l}^{\prime}+\mathbf{k}_{1}+\mathbf{k}_{2}}{D\left(\mathbf{l}^{\prime}+\mathbf{k}_{1}+\mathbf{k}_{2}\right)}+\frac{\mathbf{l}^{\prime}-\mathbf{k}_{1}}{D\left(\mathbf{l}^{\prime}-\mathbf{k}_{1}\right)}-\frac{\mathbf{k}_{1}+\mathbf{k}_{2}}{D\left(\mathbf{k}_{1}+\mathbf{k}_{2}\right)}+\frac{\mathbf{k}_{1}}{D\left(\mathbf{k}_{1}\right)}\right)_{j}\left(\frac{\mathbf{k}_{2}+\mathbf{l}^{\prime}}{\left(\mathbf{k}_{2}+\mathbf{l}^{\prime}\right)^{2}}-\frac{\mathbf{k}_{2}}{\mathbf{k}_{2}^{2}}\right)_{l}, \\
\left(T_{i l} T_{j l}^{\prime}\right)^{(b 3)}= & -\left(\frac{\mathbf{l}+\mathbf{k}_{1}+\mathbf{k}_{2}}{D\left(\mathbf{l}+\mathbf{k}_{1}+\mathbf{k}_{2}\right)}+\frac{\mathbf{l}-\mathbf{k}_{1}}{D\left(\mathbf{l}-\mathbf{k}_{1}\right)}-\frac{\mathbf{k}_{1}+\mathbf{k}_{2}}{D\left(\mathbf{k}_{1}+\mathbf{k}_{2}\right)}+\frac{\mathbf{k}_{1}}{D\left(\mathbf{k}_{1}\right)}\right)_{i}\left(\frac{\mathbf{k}_{2}+\mathbf{l}}{\left(\mathbf{k}_{2}+\mathbf{l}\right)^{2}}-\frac{\mathbf{k}_{2}}{\mathbf{k}_{2}^{2}}\right)_{l} \\
& \cdot\left(\frac{\mathbf{l}^{\prime}-\mathbf{k}_{1}-\mathbf{k}_{2}}{D\left(\mathbf{l}^{\prime}-\mathbf{k}_{1}-\mathbf{k}_{2}\right)}+\frac{\mathbf{l}^{\prime}+\mathbf{k}_{1}}{D\left(\mathbf{l}+\mathbf{k}_{1}\right)}-\frac{\mathbf{k}_{1}+\mathbf{k}_{2}}{D\left(\mathbf{k}_{1}+\mathbf{k}_{2}\right)}+\frac{\mathbf{k}_{1}}{D\left(\mathbf{k}_{1}\right)}\right)_{j}\left(\frac{\mathbf{k}_{2}-\mathbf{l}^{\prime}}{\left(\mathbf{k}_{2}-\mathbf{l}^{\prime}\right)^{2}}-\frac{\mathbf{k}_{2}}{\mathbf{k}_{2}^{2}}\right)_{l},(2
\end{aligned}
$$

and

$$
\begin{aligned}
\left(T_{i l} T_{j l}^{\prime}\right)^{(b 4)} & =\left(\frac{\mathbf{l}-\mathbf{k}_{1}-\mathbf{k}_{2}}{D\left(\mathbf{l}-\mathbf{k}_{1}-\mathbf{k}_{2}\right)}+\frac{\mathbf{l}+\mathbf{k}_{1}}{D\left(\mathbf{l}+\mathbf{k}_{1}\right)}-\frac{\mathbf{k}_{1}+\mathbf{k}_{2}}{D\left(\mathbf{k}_{1}+\mathbf{k}_{2}\right)}+\frac{\mathbf{k}_{1}}{D\left(\mathbf{k}_{1}\right)}\right)_{i}\left(\frac{\mathbf{k}_{2}-\mathbf{l}}{\left(\mathbf{k}_{2}-\mathbf{l}\right)^{2}}-\frac{\mathbf{k}_{2}}{\mathbf{k}_{2}^{2}}\right)_{l} \\
& \cdot\left(\frac{\mathbf{l}^{\prime}-\mathbf{k}_{1}-\mathbf{k}_{2}}{D\left(\mathbf{l}^{\prime}-\mathbf{k}_{1}-\mathbf{k}_{2}\right)}+\frac{\mathbf{l}^{\prime}+\mathbf{k}_{1}}{D\left(\mathbf{l}^{\prime}+\mathbf{k}_{1}\right)}-\frac{\mathbf{k}_{1}+\mathbf{k}_{2}}{D\left(\mathbf{k}_{1}+\mathbf{k}_{2}\right)}+\frac{\mathbf{k}_{1}}{D\left(\mathbf{k}_{1}\right)}\right)_{j}\left(\frac{\mathbf{k}_{2}-\mathbf{l}^{\prime}}{\left(\mathbf{k}_{2}-\mathbf{l}^{\prime}\right)^{2}}-\frac{\mathbf{k}_{2}}{\mathbf{k}_{2}^{2}}\right)_{l}
\end{aligned}
$$

The third group in Fig. ⿶ 4 c consists of diagrams with three gluons being attached to the quark loop. We obtain:

$$
\begin{aligned}
\left(T_{i l} T_{j l}^{\prime}\right)^{(c 1)}= & 2\left(\frac{\mathbf{k}_{1}+\mathbf{k}_{2}}{D\left(\mathbf{k}_{1}+\mathbf{k}_{2}\right)}-\frac{\mathbf{k}_{1}}{D\left(\mathbf{k}_{1}\right)}\right)_{i}\left(\frac{\mathbf{k}_{2}+\mathbf{l}}{\left(\mathbf{k}_{2}+\mathbf{l}\right)^{2}}-\frac{\mathbf{k}_{2}}{\mathbf{k}_{2}^{2}}\right)_{l} \\
& \left(\frac{\mathbf{l}^{\prime}+\mathbf{k}_{1}+\mathbf{k}_{2}}{D\left(\mathbf{l}^{\prime}+\mathbf{k}_{1}+\mathbf{k}_{2}\right)}+\frac{\mathbf{l}^{\prime}-\mathbf{k}_{1}}{D\left(\mathbf{l}^{\prime}-\mathbf{k}_{1}\right)}-\frac{\mathbf{k}_{1}+\mathbf{k}_{2}}{D\left(\mathbf{k}_{1}+\mathbf{k}_{2}\right)}+\frac{\mathbf{k}_{1}}{D\left(\mathbf{k}_{1}\right)}\right)_{j}\left(\frac{\mathbf{k}_{2}+\mathbf{l}^{\prime}}{\left(\mathbf{k}_{2}+\mathbf{l}^{\prime}\right)^{2}}-\frac{\mathbf{k}_{2}}{\mathbf{k}_{2}^{2}}\right)_{l} \\
\left(T_{i l} T_{j l}^{\prime}\right)^{(c 2)}= & -2\left(\frac{\mathbf{k}_{1}+\mathbf{k}_{2}}{D\left(\mathbf{k}_{1}+\mathbf{k}_{2}\right)}-\frac{\mathbf{k}_{1}}{D\left(\mathbf{k}_{1}\right)}\right)_{i}\left(\frac{\mathbf{k}_{2}+\mathbf{l}}{\left(\mathbf{k}_{2}+\mathbf{l}\right)^{2}}-\frac{\mathbf{k}_{2}}{\mathbf{k}_{2}^{2}}\right)_{l} \\
& \cdot\left(\frac{\mathbf{l}^{\prime}-\mathbf{k}_{1}-\mathbf{k}_{2}}{D\left(\mathbf{l}^{\prime}-\mathbf{k}_{1}-\mathbf{k}_{2}\right)}+\frac{\mathbf{l}^{\prime}+\mathbf{k}_{1}}{D\left(\mathbf{l}^{\prime}+\mathbf{k}_{1}\right)}-\frac{\mathbf{k}_{1}+\mathbf{k}_{2}}{D\left(\mathbf{k}_{1}+\mathbf{k}_{2}\right)}+\frac{\mathbf{k}_{1}}{D\left(\mathbf{k}_{1}\right)}\right)_{j}\left(\frac{\mathbf{k}_{2}-\mathbf{l}^{\prime}}{\left(\mathbf{k}_{2}-\mathbf{l}^{\prime}\right)^{2}}-\frac{\mathbf{k}_{2}}{\mathbf{k}_{2}^{2}}\right)_{l} \\
\left(T_{i l} T_{j l}^{\prime}\right)^{(c 3)=} & 2\left(\frac{\mathbf{l}+\mathbf{k}_{1}+\mathbf{k}_{2}}{D\left(\mathbf{l}+\mathbf{k}_{1}+\mathbf{k}_{2}\right)}+\frac{\mathbf{l}-\mathbf{k}_{1}}{D\left(\mathbf{l}-\mathbf{k}_{1}\right)}-\frac{\mathbf{k}_{1}+\mathbf{k}_{2}}{D\left(\mathbf{k}_{1}+\mathbf{k}_{2}\right)}+\frac{\mathbf{k}_{1}}{D\left(\mathbf{k}_{1}\right)}\right)_{i}\left(\frac{\mathbf{k}_{2}+\mathbf{l}}{\left(\mathbf{k}_{2}+\mathbf{l}\right)^{2}}-\frac{\mathbf{k}_{2}}{\mathbf{k}_{2}^{2}}\right)_{l} \\
& \cdot\left(\frac{\mathbf{k}_{1}+\mathbf{k}_{2}}{D\left(\mathbf{k}_{1}+\mathbf{k}_{2}\right)}-\frac{\mathbf{k}_{1}}{D\left(\mathbf{k}_{1}\right)}\right)_{j}\left(\frac{\mathbf{k}_{2}+\mathbf{l}^{\prime}}{\left(\mathbf{k}_{2}+\mathbf{l}^{\prime}\right)^{2}}-\frac{\mathbf{k}_{2}}{\mathbf{k}_{2}^{2}}\right)_{l}
\end{aligned}
$$




$$
\begin{aligned}
\left(T_{i l} T_{j l}^{\prime}\right)^{(c 4)}=-2 & \left(\frac{\mathbf{l}-\mathbf{k}_{1}-\mathbf{k}_{2}}{D\left(\mathbf{l}-\mathbf{k}_{1}-\mathbf{k}_{2}\right)}+\frac{\mathbf{l}+\mathbf{k}_{1}}{D\left(\mathbf{l}+\mathbf{k}_{1}\right)}-\frac{\mathbf{k}_{1}+\mathbf{k}_{2}}{D\left(\mathbf{k}_{1}+\mathbf{k}_{2}\right)}+\frac{\mathbf{k}_{1}}{D\left(\mathbf{k}_{1}\right)}\right)_{i}\left(\frac{\mathbf{k}_{2}-\mathbf{l}}{\left(\mathbf{k}_{2}-\mathbf{l}\right)^{2}}-\frac{\mathbf{k}_{2}}{\mathbf{k}_{2}^{2}}\right)_{l} \\
& \cdot\left(\frac{\mathbf{k}_{1}+\mathbf{k}_{2}}{D\left(\mathbf{k}_{1}+\mathbf{k}_{2}\right)}-\frac{\mathbf{k}_{1}}{D\left(\mathbf{k}_{1}\right)}\right)_{j}\left(\frac{\mathbf{k}_{2}+\mathbf{l}^{\prime}}{\left(\mathbf{k}_{2}+\mathbf{l}^{\prime}\right)^{2}}-\frac{\mathbf{k}_{2}}{\mathbf{k}_{2}^{2}}\right)_{l}
\end{aligned}
$$

Combining all contributions (a) - (c) we arrive at our final result. The sum of the products $T_{i l} T_{j l}^{\prime}$ can be written as the square of two identical (up to the primed $l$-integral) expressions:

$$
\begin{gathered}
T_{i l}=\left(\frac{\mathbf{l}+\mathbf{k}_{1}+\mathbf{k}_{2}}{D\left(\mathbf{l}+\mathbf{k}_{1}+\mathbf{k}_{2}\right)}+\frac{\mathbf{k}_{1}+\mathbf{k}_{2}}{D\left(\mathbf{k}_{1}+\mathbf{k}_{2}\right)}-\frac{\mathbf{k}_{1}-\mathbf{l}}{D\left(\mathbf{k}_{1}-\mathbf{l}\right)}-\frac{\mathbf{k}_{1}}{D\left(\mathbf{k}_{1}\right)}\right)_{i}\left(\frac{\mathbf{l}+\mathbf{k}_{2}}{\left(\mathbf{l}+\mathbf{k}_{2}\right)^{2}}-\frac{\mathbf{k}_{2}}{\mathbf{k}_{2}^{2}}\right)_{l} . \\
+(\mathbf{l} \rightarrow-\mathbf{l})
\end{gathered} .
$$

In order to obtain $M_{l}$, we simply drop the numerators in the first factor (with subscript ' $i^{6}$ ) in eq. (2.38):

$$
\begin{gathered}
T_{l}=\left(\frac{1}{D\left(\mathbf{l}+\mathbf{k}_{1}+\mathbf{k}_{2}\right)}+\frac{1}{D\left(\mathbf{k}_{1}+\mathbf{k}_{2}\right)}-\frac{1}{D\left(\mathbf{k}_{1}-\mathbf{l}\right)}-\frac{1}{D\left(\mathbf{k}_{1}\right)}\right)\left(\frac{\mathbf{l}+\mathbf{k}_{2}}{\left(\mathbf{l}+\mathbf{k}_{2}\right)^{2}}-\frac{\mathbf{k}_{2}}{\mathbf{k}_{2}^{2}}\right)_{l} \\
+(\mathbf{l} \rightarrow-\mathbf{l})
\end{gathered}
$$

which defines $T_{l}$, and $M_{l}$ then follows from eq. (2.24). Inserting these results into eq. (2.12) eq. (2.15), we arrive at our final expression for the ep cross section.

\section{Impact Parameter Representation}

It is very instructive to analyze the structure of the matrix element $M_{i l}$ or $M_{l}$ in impact parameter space. As will be shown below the simplification in impact parameter space arises in form of two wave functions of which the first describes the dissociation of the photon into the $q \bar{q}$ - pair and the second the subsequent radiation of the gluon from the quark or the antiquark. The couplings of the $t$ - channel gluons generate phase - factors which after some regrouping can be turned into effective dipole cross sections. For the numerical analysis later on we have, of course, to use the momentum representation.

We start by taking the Fourier transformation of $T_{i l}$ :

$$
\begin{aligned}
& \tilde{T}_{i l}= \int d^{2} \mathbf{k}_{1} d^{2} \mathbf{k}_{2} \mathrm{e}^{i \mathbf{k}_{1} \cdot \mathbf{r}_{1}+i \mathbf{k}_{2} \cdot \mathbf{r}_{2}} T_{i l} \\
&= \Psi_{i}^{T}\left(\mathbf{r}_{1}\right) \int d^{2} \mathbf{k}_{2} \mathrm{e}^{i \mathbf{k}_{2} \cdot \mathbf{r}_{2}}\left[\left\{\mathrm{e}^{-i\left(\mathbf{k}_{2}+\mathbf{l}\right) \cdot \mathbf{r}_{1}}+\mathrm{e}^{-i \mathbf{k}_{2} \cdot \mathbf{r}_{1}}-\mathrm{e}^{-i \mathbf{l} \cdot \mathbf{r}_{1}}-1\right\}\left\{\frac{\mathbf{k}_{2}+\mathbf{l}}{\left(\mathbf{k}_{2}+\mathbf{l}\right)^{2}}-\frac{\mathbf{k}_{2}}{\mathbf{k}_{2}^{2}}\right\}_{l}\right. \\
&\quad+(\mathbf{l} \rightarrow-\mathbf{l})] \\
&=\Psi_{i}^{T}\left(\mathbf{r}_{1}\right)\left[\varphi_{l}\left(\mathbf{r}_{2}-\mathbf{r}_{1}\right)\left\{\mathrm{e}^{-i l \cdot \mathbf{r}_{2}}+\mathrm{e}^{-i l \cdot\left(\mathbf{r}_{2}-\mathbf{r}_{1}\right)}-\mathrm{e}^{-i l \cdot \mathbf{r}_{1}}-1\right\}+(\mathbf{l} \rightarrow-\mathbf{l})\right. \\
&\left.\quad-\varphi_{l}\left(\mathbf{r}_{2}\right)\left\{\mathrm{e}^{-i l \cdot \mathbf{r}_{2}}+\mathrm{e}^{-i l \cdot\left(\mathbf{r}_{2}-\mathbf{r}_{1}\right)}-\mathrm{e}^{i l \cdot \mathbf{r}_{1}}-1\right\}+(\mathbf{l} \rightarrow-\mathbf{l})\right]
\end{aligned}
$$

where $\Psi^{T}$ and $\varphi$ are defined as

$$
\begin{aligned}
\Psi_{i}^{T}(\mathbf{r}) & =\int d^{2} \mathbf{k} \mathrm{e}^{i \mathbf{k} \cdot \mathbf{r}} \frac{\mathbf{k}_{i}}{D(\mathbf{k})}=-2 \pi i \frac{\mathbf{r}_{i}}{|\mathbf{r}|} \sqrt{\alpha(1-\alpha) Q^{2}} \mathrm{~K}_{1}\left(\sqrt{\alpha(1-\alpha) Q^{2} r^{2}}\right) \\
\varphi_{l}(\mathbf{r}) & =\int d^{2} \mathbf{k} \mathrm{e}^{i \mathbf{k} \cdot \mathbf{r}} \frac{\mathbf{k}_{l}}{\mathbf{k}^{2}}=-2 \pi i \frac{\mathbf{r}_{l}}{|\mathbf{r}|^{2}}
\end{aligned}
$$


If we now introduce the effective dipole cross-section as

$$
\sigma(\mathbf{r})=\int \frac{d^{2} l}{\pi l^{2}} \mathcal{F}\left(x_{\mathbb{P}}, l^{2}\right)\left(1-\mathrm{e}^{i l \cdot \mathbf{r}}\right)\left(1-\mathrm{e}^{-i l \cdot \mathbf{r}}\right)
$$

we finally obtain for $\tilde{M}_{i l}$, the Fourier transform of $M_{i l}$ :

$$
\tilde{M}_{i l}=\Psi_{i}^{T}\left(\mathbf{r}_{1}\right)\left\{\varphi_{l}\left(\mathbf{r}_{2}\right)-\varphi_{l}\left(\mathbf{r}_{2}-\mathbf{r}_{1}\right)\right\}_{l}\left[\sigma\left(\mathbf{r}_{2}\right)+\sigma\left(\mathbf{r}_{2}-\mathbf{r}_{1}\right)-\sigma\left(\mathbf{r}_{1}\right)\right] .
$$

The vector $\mathbf{r}_{1}$ denotes the separation of the quark and antiquark, $\mathbf{r}_{2}$ the separation of the quark and gluon and $\mathbf{r}_{2}-\mathbf{r}_{1}$ the separation of the antiquark and gluon. The result in eq. (3.4) is the same as in ref. 18]. One derives the corresponding expression for $\tilde{M}_{l}$ by substituting the transverse wave function $\Psi^{T}$ by the longitudinal wave function $\Psi^{L}$,

$$
\Psi^{L}(\mathbf{r})=\int d^{2} \mathbf{k} \mathrm{e}^{i \mathbf{k} \cdot \mathbf{r}} \frac{1}{D(\mathbf{k})}=2 \pi \mathrm{K}_{0}\left(\sqrt{\alpha(1-\alpha) Q^{2} r^{2}}\right) .
$$

The impact parameter representation suggests the intuitive interpretation that following the initial dissociation of the photon, which is described by $\Psi^{T, L}$, each of the quarks radiates off a gluon represented by $\varphi$. After the creation of the $q \bar{q} g$-state the interaction with the target processes pairwise, i.e. each of the pairs $q \bar{q}, q g$ and $\bar{q} g$ gives a separate contribution as indicated by the different arguments of the effective dipole cross-section $\sigma$. As was pointed out in ref. [18], however, the color structure is not quite consistent with the previous interpretation. In particular the interaction of the $q \bar{q}$ as part of the $q \bar{q} g$-final state is color suppressed by powers of $N_{c}$. The reason for having a non-suppressed contribution from the $q \bar{q}$ - pair is hidden in the requirement to include the interaction of the 'renormalized' $q \bar{q}$ - pair [18]. In other words, the expression in eq. (3.4) also includes a contribution where the interaction with the $q \bar{q}$ takes place before a gluon is emitted. This contribution is not color suppressed. The result eq. (3.4) has been derived from Feynman diagrams which automatically take into account all necessary configurations.

Taking the square of the amplitude in impact parameter space one finds

$$
\tilde{M}_{i l} \tilde{M}_{i l}=(2 \pi)^{2}\left|\Psi^{T}\left(\mathbf{r}_{1}\right)\right|^{2} \frac{\mathbf{r}_{1}^{2}}{\mathbf{r}_{2}^{2}\left(\mathbf{r}_{2}-\mathbf{r}_{1}\right)^{2}}\left[\sigma\left(\mathbf{r}_{2}\right)+\sigma\left(\mathbf{r}_{2}-\mathbf{r}_{1}\right)-\sigma\left(\mathbf{r}_{1}\right)\right]^{2} .
$$

The expression $\frac{\mathbf{r}_{1}^{2}}{\mathbf{r}_{2}^{2}\left(\mathbf{r}_{2}-\mathbf{r}_{1}\right)^{2}}$ is characteristic for the dipole formalism [19]. The exact equivalence of eq. (3.6) and the corresponding double - dipole scattering still needs to be established.

Assuming strong ordering in the separation of the quark - antiquark - pair and the separation of the quarks and the gluon, $\left|\mathbf{r}_{1}\right| \ll\left|\mathbf{r}_{2}\right|$, one obtains the simple factorized form

$$
\tilde{M}_{i l} \tilde{M}_{i l}=(2 \pi)^{2} \mathbf{r}_{1}^{2}\left|\Psi^{T}\left(\mathbf{r}_{1}\right)\right|^{2} 4 \frac{\sigma^{2}\left(\mathbf{r}_{2}\right)}{\mathbf{r}_{2}^{4}}
$$

\section{A Few Special Kinematic Regions}

In this section we consider a few kinematical regions of interest for which analytic expressions can be obtained. We will average over azimuthal angles. As a result, we need to consider only eq. (2.12) and eq. (2.14). Eq. (2.15) would require a discussion similar to eq.(2.14).

First we study the 'hard' region:

$$
Q_{0}^{2} \ll \mathbf{l}^{2} \ll \mathbf{k}_{2}^{2} \sim D\left(\mathbf{k}_{1}\right) \sim D\left(\mathbf{k}_{1}+\mathbf{k}_{2}\right) \sim \tilde{\mathbf{k}}^{2}
$$


where $Q_{0}^{2}$ denotes a hadronic scale which separates the hard and the soft region. For simplicity we assume that all relevant scales in the problem are of the same order of magnitude, $\tilde{k}^{2}$. In the $\gamma^{*}-\mathbb{P}$ CM-system the gluon jet is close to the direction of the Pomeron, and the two quarks form a broader jet in the opposite direction. For $T_{i l}$ in eq. (2.38) we find (after averaging over the azimuthal angle of $\mathbf{l}$ ):

$T_{i l}=\frac{\mathbf{l}^{2}}{\mathbf{k}_{2}^{2}}\left(\frac{\delta_{i m}}{D\left(\mathbf{k}_{1}+\mathbf{k}_{2}\right)}-2 \frac{\left(\mathbf{k}_{1}+\mathbf{k}_{2}\right)_{i}\left(\mathbf{k}_{1}+\mathbf{k}_{2}\right)_{m}}{D^{2}\left(\mathbf{k}_{1}+\mathbf{k}_{2}\right)}+\frac{\delta_{i m}}{D\left(\mathbf{k}_{1}\right)}-2 \frac{\left(\mathbf{k}_{1}\right)_{i}\left(\mathbf{k}_{1}\right)_{m}}{D^{2}\left(\mathbf{k}_{1}\right)}\right)\left(\delta_{m l}-2 \frac{\left(\mathbf{k}_{2}\right)_{m}\left(\mathbf{k}_{2}\right)_{l}}{\mathbf{k}_{2}^{2}}\right)($

Using eq. (2.24) and eq. (2.25) we obtain for $M_{i l} M_{i l}^{\prime}$ :

$$
\begin{aligned}
M_{i l} M_{i l}^{\prime}= & \frac{2}{\left(\mathbf{k}_{2}^{2}\right)^{2}}\left\{\left(\frac{1}{D\left(\mathbf{k}_{1}+\mathbf{k}_{2}\right)}+\frac{1}{D\left(\mathbf{k}_{1}\right)}\right)^{2}\right. \\
& -2\left(\frac{1}{D\left(\mathbf{k}_{1}+\mathbf{k}_{2}\right)}+\frac{1}{D\left(\mathbf{k}_{1}\right)}\right)\left(\frac{\left(\mathbf{k}_{1}+\mathbf{k}_{2}\right)^{2}}{D^{2}\left(\mathbf{k}_{1}+\mathbf{k}_{2}\right)}+\frac{\mathbf{k}_{1}^{2}}{D^{2}\left(\mathbf{k}_{1}\right)}\right) \\
& \left.+2\left(\frac{\left(\mathbf{k}_{1}+\mathbf{k}_{2}\right)^{2}}{D^{2}\left(\mathbf{k}_{1}+\mathbf{k}_{2}\right)}+\frac{\mathbf{k}_{1}^{2}}{D^{2}\left(\mathbf{k}_{1}\right)}\right)^{2}+4 \frac{\left[\mathbf{k}_{1} \cdot\left(\mathbf{k}_{1}+\mathbf{k}_{2}\right)\right]^{2}-\mathbf{k}_{1}^{2}\left(\mathbf{k}_{1}+\mathbf{k}_{2}\right)^{2}}{D^{2}\left(\mathbf{k}_{1}+\mathbf{k}_{2}\right) D^{2}\left(\mathbf{k}_{1}\right)}\right\} \cdot\left[x_{\mathbb{P}} g\left(x_{\mathbb{P}}, \tilde{\mathbf{k}}^{2}\right)\right]^{2}
\end{aligned}
$$

The transverse cross section is obtained by inserting eq. (4.3) into eq. (2.12). The most remarkable feature of this result is the appearance of the square of the gluon structure function: because of the prefactor $1 /\left(\mathbf{k}_{2}^{2}\right)^{2}$, one expects the 'hard' region of large $\mathbf{k}_{2}^{2}$ to be suppressed. But as a result of its rise at small $x_{\mathbb{P}}$, the gluon structure function provides an enhancement factor of the hard region which increases with decreasing $x_{\mathbb{P}}$.

As a special case of the hard region we consider the case of strong ordering of the transverse momenta, $\mathbf{k}_{2}^{2} \ll \mathbf{k}_{1}^{2}$. Then eq. (4.3) simplifies into

$$
T_{i l}=2 \frac{\mathbf{l}^{2}}{\mathbf{k}_{2}^{2}}\left(\frac{\delta_{i m}}{D\left(\mathbf{k}_{1}\right)}-2 \frac{\left(\mathbf{k}_{1}\right)_{i}\left(\mathbf{k}_{1}\right)_{m}}{D^{2}\left(\mathbf{k}_{1}\right)}\right)\left(\delta_{m l}-2 \frac{\left(\mathbf{k}_{2}\right)_{m}\left(\mathbf{k}_{2}\right)_{l}}{\mathbf{k}_{2}^{2}}\right)
$$

With the approximation of eq. (2.19) we obtain for $M_{i l} M_{i l}^{\prime}$ :

$$
M_{i l} M_{i l}^{\prime}=8 \frac{Q^{4}+m^{4}}{\left(Q^{2}+m^{2}\right)^{4}}\left(\frac{m^{2}}{\mathbf{k}_{1}^{2}}\right)^{2} \frac{1}{\left(\mathbf{k}_{2}^{2}\right)^{2}}\left[x_{\mathbb{P}} g\left(x_{\mathbb{P}}, \mathbf{k}_{2}^{2}\right)\right]^{2}
$$

It might be interesting to note that an early approach 20] to diffractive deep inelastic scattering which not only considers the radiation of one but many gluons was based on the special case discussed above. An extension of these results towards large $\beta$ can be found in ref. [21] which in our notation reads

$$
M_{i l} M_{i l}^{\prime}=8 \frac{Q^{4}+m^{4}}{\left(Q^{2}+m^{2}\right)^{4}}\left(\frac{m^{2}}{\mathbf{k}_{1}^{2}}\right)^{2}\left(\frac{M^{2}-m^{2}}{M^{2}+Q^{2}}\right)^{4}\left(\frac{M^{2}+2 m^{2}+3 Q^{2}}{M^{2}+Q^{2}}\right)^{2} \frac{1}{\left(\mathbf{k}_{2}^{2}\right)^{2}}\left[x_{\mathbb{P}} g\left(x_{\mathbb{P}}, \mathbf{k}_{2}^{2}\right)\right]^{2} .
$$

The main observation here is the strong suppression of $M_{i l} M_{i l}^{\prime}$ when $m^{2}$ becomes of the order of $M^{2}$. This fact is also implemented in the parameterization of [3].

Next we say a few more words about the small- $\mathbf{k}_{2}^{2}$ region. As we have noted already after eq. (4.3), the region of small $\mathbf{k}_{2}^{2}$ is expected to give an important contribution to the integrated diffractive cross section. On the other hand, if $\mathbf{k}_{2}^{2}$ becomes smaller than, say, the hadronic scale 
$Q_{0}^{2}$, perturbation becomes unreliable and we cannot apply our formulae. For practical purposes, however, it would be attractive to find an extrapolation of our jet cross section into the region where the gluon jets becomes soft. Following the discussion of the $q \bar{q}$ final state in diffraction dissociation in [6] we shall present an extrapolation of our perturbative calculation, which at small $\mathbf{k}_{2}^{2}$ leads to the Ingelman-Schlein picture of the Pomeron structure function. First, at small $\mathbf{k}_{2}^{2}$, the approximation $\mathbf{l}^{2} \ll \mathbf{k}_{2}^{2}$ will no longer be valid; $\mathbf{l}^{2}$ can become of the same order as or even larger than $\mathbf{k}_{2}^{2}$. For simplicity we do not consider the most general case but still retain the approximation $\mathbf{l}^{2}, \mathbf{k}_{2}^{2} \ll \mathbf{k}_{1}^{2}$. After integrating over the azimuthal angle of $\mathbf{l}$ we find for $T_{i l}$ :

$$
T_{i l}=2\left(\frac{\delta_{i m}}{D\left(\mathbf{k}_{1}\right)}-2 \frac{\left(\mathbf{k}_{1}\right)_{i}\left(\mathbf{k}_{1}\right)_{m}}{D^{2}\left(\mathbf{k}_{1}\right)}\right)\left(\delta_{m l}-2 \frac{\left(\mathbf{k}_{2}\right)_{m}\left(\mathbf{k}_{2}\right)_{l}}{\mathbf{k}_{2}^{2}}\right)\left(\Theta\left(\mathbf{l}^{2}-\mathbf{k}_{2}^{2}\right)+\frac{\mathbf{l}^{2}}{\mathbf{k}_{2}^{2}} \Theta\left(\mathbf{k}_{2}^{2}-\mathbf{l}^{2}\right)\right)
$$

which is consistent with the result found earlier in ref. [11]. Before inserting eq. (4.6) into eq. (2.29), we have to discuss the two gluon amplitude $\mathcal{F}\left(x_{\mathbb{P}}, 1^{2}\right)$. As long as the momentum scale $l^{2}$ at the upper end is large, it can (to a good approximation) be identified with the unintegrated gluon structure function (see eq. (2.25)). Now we will try to find an extrapolation into the soft $\mathbf{k}_{2}^{2}$ region which, at low $\mathbf{k}_{2}^{2}$, smoothly turns into the Pomeron structure function picture. An easy way has been sketched in [6], and here we outline the argument in somewhat more detail. We make the following simple double Mellin transform ansatz for $\mathcal{F}$ :

$$
\mathcal{F}\left(x_{\mathbb{P}}, \mathbf{l}^{2}\right)=\phi_{0} \frac{1}{Q_{0}^{2}} \int \frac{d \omega}{2 \pi i} \int \frac{d \mu}{2 \pi i}\left(\frac{1}{x_{\mathbb{P}}}\right)^{\omega}\left(\frac{\mathbf{l}^{2}}{Q_{0}^{2}}\right)^{\mu} \frac{1}{\omega-\chi(\mu)}
$$

where the integration contours run along the imaginary axis $(-1<\operatorname{Re} \mu<0, \chi(\mu)<\operatorname{Re\omega })$, and $\phi_{0}$ denotes the overall normalization constant. If we would perform the $\mu$ integral first by picking up a pole at $\mu=\chi^{-1}(\omega)=\gamma(\omega)$, we obtain the anomalous dimension. However, here it is more convenient to model the function $\chi(\mu)$ and to do first the $\omega$ integral. Let us assume that $\chi(\mu)$ is positive and becomes large both at $\mu=-1$ and $\mu=0$ (similar to the BFKL characteristic function). We then obtain:

$$
\mathcal{F}\left(x_{\mathbb{P}}, \mathbf{l}^{2}\right)=\phi_{0} \frac{1}{Q_{0}^{2}} \int \frac{d \mu}{2 \pi i} \exp \left(\chi(\mu) \log \frac{1}{x_{\mathbb{P}}}+\mu \log \frac{\mathbf{l}^{2}}{Q_{0}^{2}}\right)
$$

Combining this ansatz with eq. (4.6) we write the $l^{2}$ integral as:

$$
\left(\frac{\mathbf{k}_{2}^{2}}{Q_{0}^{2}}\right)^{\mu} \int^{\mathbf{k}_{1}^{2} / \mathbf{k}_{2}^{2}} \frac{d \mathbf{l}^{\prime 2}}{\mathbf{l}^{\prime 2}}\left(\mathbf{l}^{\prime 2}\right)^{\mu}\left[\Theta\left(\mathbf{l}^{\prime 2}-1\right)+\mathbf{l}^{\prime 2} \Theta\left(1-\mathbf{l}^{\prime 2}\right)\right]
$$

Inserting the $\mathbf{k}_{2}^{2}$-dependent prefactor of eq. (4.9) into the $\mu$ integral of eq. (4.7) and performing a stationary phase analysis, we find that for $Q_{0}^{2} \ll \mathbf{k}_{2}^{2}$ the stationary point moves towards $\mu=-1$, whereas for the opposite case $\mathbf{k}_{2}^{2} \ll Q_{0}^{2}$ the stationary point is near $\mu=0$. In order to evaluate the $\mathrm{l}^{\prime 2}$ integral in eq. (4.8) near $\mu=-1$ or $\mu=0$ we have to specify the lower limit of integration. In the first case (large $\mathbf{k}_{2}^{2}, \mu \rightarrow-1$ ), the first term in eq. (4.9) stays constant whereas the second part gives a $\log \mathbf{k}_{2}^{2} / Q_{0}^{2}$ from the region $Q_{0}^{2} / \mathbf{k}_{2}^{2}<\mathbf{l}^{\prime 2}<1$. In the second case (small $\mathbf{k}_{2}^{2}, \mu \rightarrow 0$ ) the first term gives a logarithmic enhancement $\log \mathbf{k}_{1}^{2} / Q_{0}^{2}$ whereas the second term stays constant (which is equal to 1 if the $l^{\prime 2}$ integral extends down to 0 ). As a result we obtain for the $\mathbf{l}^{2}$ integral:

$$
\begin{aligned}
\Phi\left(\mathbf{k}_{1}^{2}, \mathbf{k}_{2}^{2}, x_{\mathbb{P}}\right) & =\int^{\mathbf{k}_{1}^{2}} \frac{d \mathbf{l}^{2}}{\mathbf{l}^{2}} \mathcal{F}\left(x_{\mathbb{P}}, \mathbf{l}^{2}\right)\left(\Theta\left(\mathbf{l}^{2}-\mathbf{k}_{2}^{2}\right)+\frac{\mathbf{l}^{2}}{\mathbf{k}_{2}^{2}} \Theta\left(\mathbf{k}_{2}^{2}-\mathbf{l}^{2}\right)\right) \\
& \sim\left\{\begin{array}{ccc}
\phi_{0} / \mathbf{k}_{2}^{2} \ln \left(\mathbf{k}_{2}^{2} / Q_{0}^{2}\right) & \mathbf{k}_{2}^{2} \gg Q_{0}^{2} & \mu \sim-1 \\
\phi_{0} / Q_{0}^{2} \ln \left(\mathbf{k}_{1}^{2} / \mathbf{k}_{2}^{2}\right) & \mathbf{k}_{2}^{2} \ll Q_{0}^{2} & \mu \sim 0
\end{array}\right\}
\end{aligned}
$$


Together with eq. (4.6), we obtain for $M_{i l} M_{i l}^{\prime}$ :

$$
M_{i l} M_{i l}^{\prime}=\Phi^{2} \frac{8 m^{4}}{\left(\mathbf{k}_{1}^{2}\right)^{2}} \frac{Q^{4}+m^{4}}{\left(Q^{2}+m^{2}\right)^{4}}
$$

Inserting this into (2.13), and making use of (2.20) we find the expected result: the transverse cross section belongs to leading twist, and the integral over $\mathbf{k}_{1}^{2}$ diverges logarithmically.

A nice feature of this extrapolation into the soft non-perturbative region is the following. As we have described above, the two cases (large and small $\mathbf{k}_{2}^{2}$ ) correspond to different regions of the $\mathbf{l}^{2}$ integral: the first case is the usual strong ordering with $Q_{0}^{2}<\mathbf{l}^{2}<\mathbf{k}_{2}^{2}$. In the second case $\mathbf{l}^{2}$ prefers to be larger than $\mathbf{k}_{2}^{2}$ : in this region we simply drop $\mathbf{k}_{2}^{2}$ in comparison with $\mathbf{l}$ and $\mathbf{k}_{1}$. As a result, the non-planar diagrams in Fig. 目 become small in comparison with the planar ones. What is left can be interpreted as a 'gluon component of the Pomeron structure function'. This has to be compared with the first case, where all diagrams are equally important, i.e. the Pomeron interacts with the whole diffractive system.

Next we consider the case where the gluon stays hard (large $\mathbf{k}_{2}$ ) and one of the quarks becomes soft, i.e. $\mathbf{k}_{1} \sim 0$ or $\mathbf{k}_{1}+\mathbf{k}_{2} \sim 0$. Let us assume $\mathbf{k}_{1}$ to be small $\left(\mathbf{k}_{1}^{2}<\mathbf{l}^{2}<Q_{0}^{2}\right)$ and go back to eq. (2.38). With $\mathbf{l}^{2}>\mathbf{k}_{1}^{2}$ only the third term in the first bracket contributes and we find after integration over the azimuth angle:

$$
T_{i l}=\left(\delta_{i l}-2 \frac{\left(\mathbf{k}_{2}\right)_{i}\left(\mathbf{k}_{2}\right)_{l}}{\mathbf{k}_{2}^{2}}\right) \frac{\mathbf{l}^{2}}{D(\mathbf{l})} .
$$

Using our ansatz for the unintegrated structure function eq. (4.7) with the soft extrapolation $\mu \rightarrow 0$ we can perform the l-integration

$$
\begin{aligned}
\Phi\left(k_{1}, x_{\mathbb{P}}\right) & =\int_{\mathbf{k}_{1}^{2}}^{Q_{0}^{2}} \frac{d \mathbf{l}^{2}}{\mathbf{l}^{2}}\left(\frac{\mathbf{l}^{2}}{Q_{0}^{2}}\right)^{\mu} \frac{\mathbf{l}^{2}}{D(\mathbf{l})} \\
& \sim \ln \left(\frac{D\left(Q_{0}\right)}{D\left(\mathbf{k}_{1}\right)}\right)
\end{aligned}
$$

and finally arrive at

$$
M_{i l} M_{i l}^{\prime}=\Phi^{2} \frac{2}{\left(\mathbf{k}_{2}^{2}\right)^{2}}
$$

It is important to note that this kinematical limit yields a contribution which breaks (collinear) factorization. The result in eq. (4.14) is similar to the 'super-hard component of the Pomeron' introduced in ref. [14]. In a scenario where the photon virtuality $Q^{2}$ is smaller than the gluon momentum $\mathbf{k}_{2}^{2}$, one would expect from the quark loop a large logarithm of the form $\log \left(\mathbf{k}_{2}^{2} / Q^{2}\right)$, if factorization worked. The potential softness of one of the quarks which controls the scale $\mathbf{l}^{2}$ in eq. (4.13) is responsible for the lack of such a hard logarithm. In other words the soft part of the process decouples from the jets in the final state. With regard to the inclusive diffractive cross section, the hard gluon jet combined with a soft quark gives only a rather small fraction of the total contribution. The leading contribution comes from the scenario discussed earlier where the gluon becomes soft and the quarks are hard. In this case we have the familiar strong ordering situation which provides a hard $\operatorname{logarithm} \log \left(Q^{2} / \mathbf{k}_{2}^{2}\right)$ in accordance with factorization for diffractive deep inelastic scattering [15] and the notion of a Pomeron structure function. Factorization is still 
violated by contributions with $\mathbf{k}_{2}^{2}$ bigger than the assumed factorization scale. Since the mean value of $\mathbf{k}_{2}^{2}$ is close to $Q_{0}^{2}$, as can be inferred from eq. (4.10) and eq. (4.11), one can argue that for any scale well above $Q_{0}^{2}$, factorization should hold.

Finally we return to our 'hardness condition' in eq. (2.2). For the gluon jet, its transverse momentum square $\mathbf{k}_{2}^{2}$ coincides with the virtuality of the vertical gluon line in Fig. \#a: for small $\mathbf{k}_{2}^{2}$ this 'Pomeron structure function' diagram becomes more important than the other non-planar diagrams; the boundary between hard and soft physics is defined by the off-shellness of the gluon emitted from the $q \bar{q}$ pair. Correspondingly, for the quark-antiquark pair it is the virtuality of the quark (or antiquark) before it emits the gluon. With the approximations discussed in the beginning of this section, the requirement that the four momentum square of this quark has to be large then leads to the last two equations in eq. (2.2).

\section{A Numerical Study}

In this section we study the specific signature of the process $e+p \rightarrow q+q+g+p^{\prime}$ with the cross section calculated in the previous sections. We work in the $\gamma-\mathbb{P}$ center of mass system, and the gluon will be restricted to the forward hemisphere of the proton (Pomeron). We have implemented the formulae of eq. (2.12) - eq. (2.17) into a full hadron level Monte Carlo program [22]. To avoid soft divergences of the matrix element we impose a cut in $|\hat{t}|=\left|\left(q-p_{q}\right)^{2}\right|>2 \mathrm{GeV}^{2}$ and $|\hat{u}|=\left|\left(q-p_{\bar{q}}\right)^{2}\right|>2 \mathrm{GeV}^{2}$, where $q, p_{q}=q+r-k_{1}-k_{2}$, and $p_{\bar{q}}=k_{1}$ are the four momenta of the photon, the upper outgoing quark and the lower outgoing antiquark (Fig. 5), respectively, and a cut on the transverse momentum squared of the gluon $\mathbf{k}_{2}^{2}>2 \mathrm{GeV}^{2}$. The coupling $\alpha_{s}$ is kept fixed at $\alpha_{s}=0.25$. For the gluon structure function we use the NLO parameterization of GRV [23]. We divide this numerical study into two parts. In order to analyze the general features of the threeparton final state we first work in an "ideal" (i.e. truly asymptotic) kinematic environment where the invariant mass $M$ is much bigger than all transverse momenta $\left(Q^{2}=100 \mathrm{GeV}^{2}, M^{2}=900\right.$ $\mathrm{GeV}^{2}$, (i.e. $\left.\beta=0.1\right), x_{\mathbb{P}}<10^{-2}$ ). In the second part we turn to HERA kinematics.

\section{$5.1 \quad$ Asymptotic region}

First we have to define the region of validity of our calculation. In deriving our cross section formulae we have made several approximations. Regge-kinematics requires that $\alpha_{2} \ll \alpha_{1}, 1-\alpha_{1}$. In our numerical analysis we therefore impose a kinematical cut and require that $\alpha_{2}<\frac{1}{5} \alpha_{1}, \frac{1}{5}\left(1-\alpha_{1}\right)$. Second, we expect that for large $M^{2}$ the mass of the $q \bar{q}$ system will be small: in order to enforce this condition we further demand that $m_{q \bar{q}}^{2}<\frac{1}{4} M^{2}$.

We begin with $p_{T}$ spectra (Fig. 6). A fit to a power-like behavior $\left(1 / p_{T}^{2}\right)^{p}$ (for $p_{T}^{2}<10 \mathrm{GeV}^{2}$ ) gives $p=0.82$ and $p=1.63$ for the quark and gluon transverse momenta, respectively. This behavior is not far from the naive estimate based upon eq. (4.5): together with the phase space factors in eq. (2.12) - eq. (2.13) one expects $p=1$ for the quark momentum $\mathbf{k}_{1}^{2}$, and $p$ slightly less than 2 for the gluon momentum $\mathbf{k}_{2}^{2}$. A very striking feature is the fact that for a rather large fraction of events the ordering condition $\mathbf{k}_{2}^{2}<\mathbf{k}_{1}^{2},\left(\mathbf{k}_{1}+\mathbf{k}_{2}\right)^{2}$ is not fulfilled: we find that only $60 \%$ of the events satisfy this condition. This demonstrates that the simple boson-gluon picture in which the gluon is emitted with a transverse momentum much smaller than that of the two quarks may be very unreliable. On the other hand, for almost all events the square of the gluon transverse momentum is less than the virtualities of the exchanged quarks $\left(\mathbf{k}_{2}^{2}<|\hat{t}|,|\hat{u}|\right)$. 

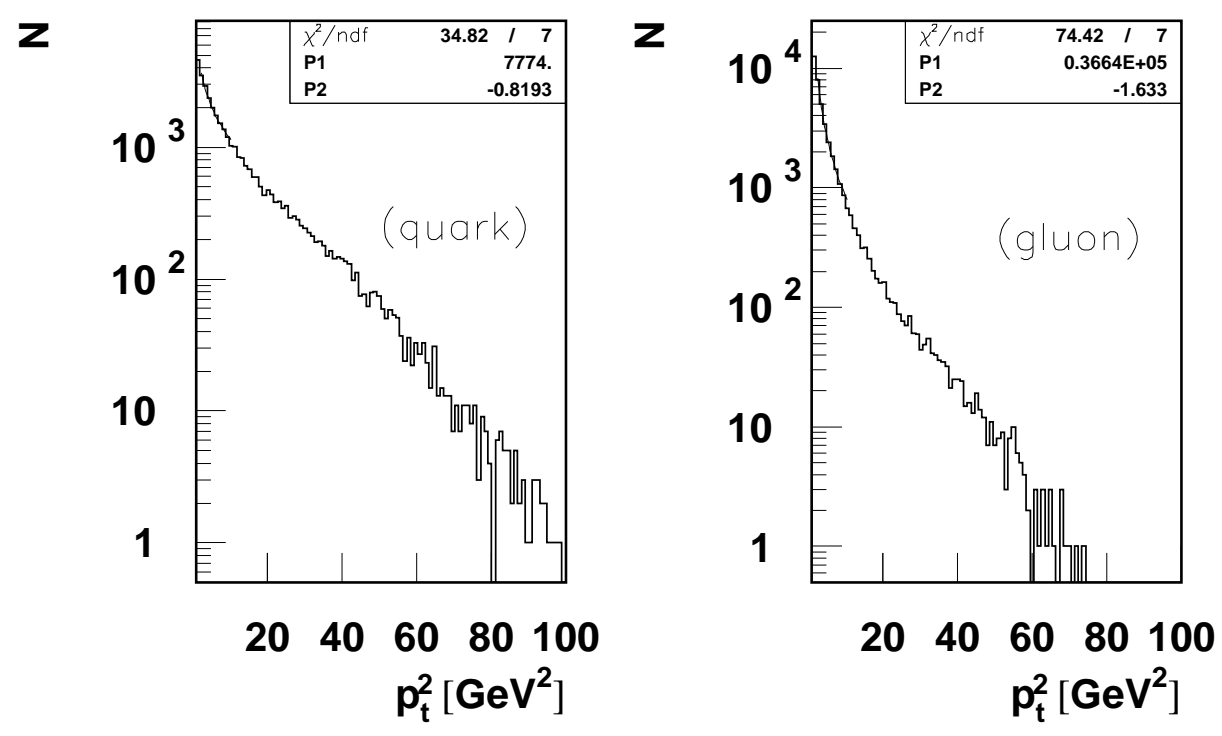

Figure 6: The distribution of transverse momenta for quarks and gluons.

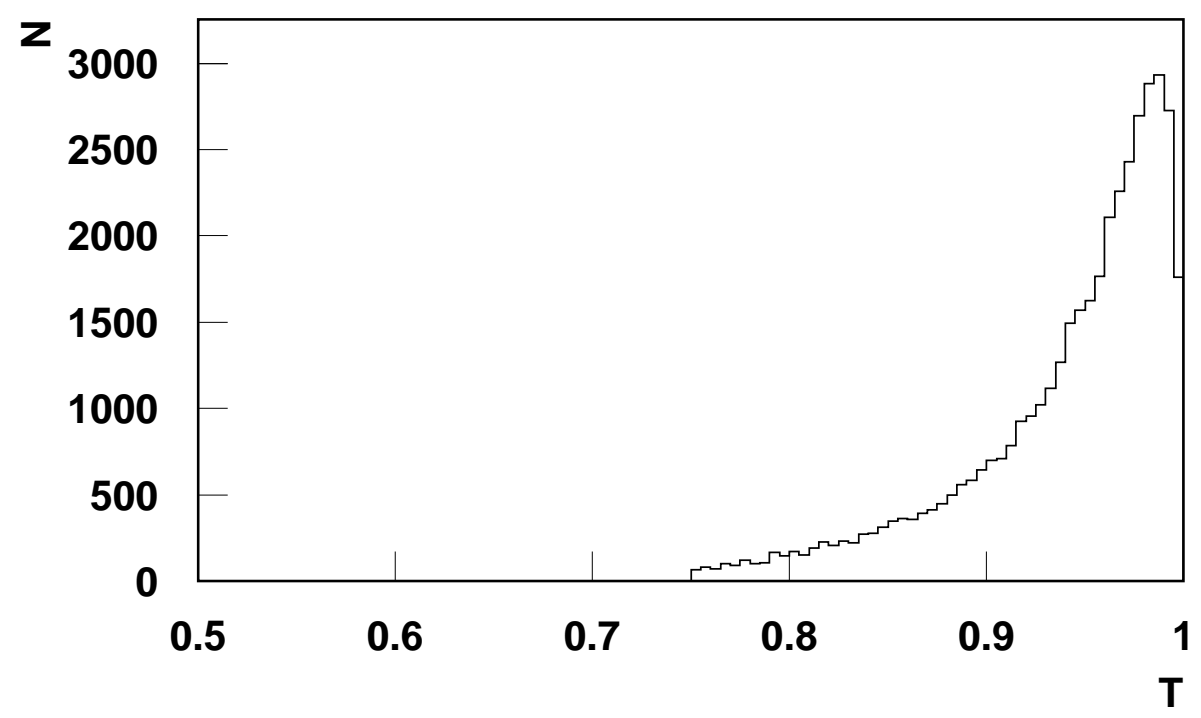

Figure 7: The distribution of thrust $T$.

Next we look into the spatial distribution of the three-parton final state. From the experimental point of view it is convenient to begin with the thrust distribution: the plot in Fig. 7 shows that a large fraction of the events has a two-jet like structure $(T>0.9)$. However, there remains also a sizable fraction (about 20\%) of three-jet events for which $T<0.9$. To analyze these samples 


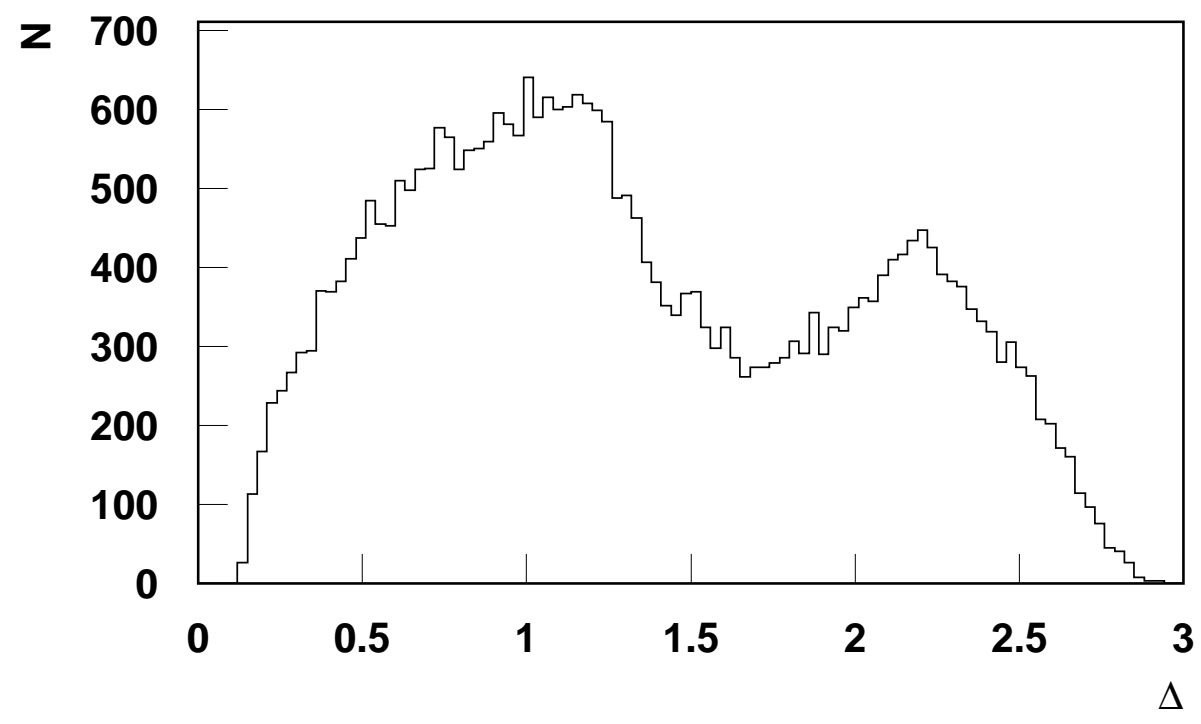

Figure 8: The distribution of the opening angle $\Delta$ of the $q \bar{q}$ pair for values of thrust $T>0.9$ (two jet configuration).

in more detail, we begin with the two-jet events and we show in Fig. 8 the distribution of the opening angle $\Delta$ of the $q \bar{q}$ pair: the distribution peaks at both small and large opening angles. We divide the events into two sets: events with large opening angle $(\Delta>\pi / 2)$ and those with small opening angle $(\Delta<\pi / 2)$. In the first case we find (Fig. 9) a large asymmetry in the longitudinal momenta and a big invariant mass of the two quarks. The quark which moves with maximum momentum $(\sim 15 \mathrm{GeV})$ in the photon direction forms one jet whereas the second quark moves into the opposite direction. The second quark and the gluon form the second jet sharing the jet momentum. In Fig. 10 a the correlation of the longitudinal quark and gluon momentum for $T>0.9$ and $\Delta>\pi / 2$ (large opening angle) is shown. The upper band contains events where the quark moves isolated in the photon direction whereas the antiquark moves along with the gluon in the Pomeron direction. The lower band contains events where the quark and the gluon form a jet sharing the jet-momentum.

For those events with small opening angle, on the other hand, the momenta of the two quarks are much more symmetric. In this case, the two quarks form a jet opposite to the gluon (Fig. 9).

In Fig. $10 b$ we show the quark and antiquark longitudinal momentum for $T>0.9$ and $\Delta<\pi / 2$ (small opening angle). Both momenta are strongly correlated.

In all these cases the jet axis (i.e. the direction of the gluon or quark) lies mainly in the Pomeron direction ("aligned gluon configuration"): Fig. 12 (full curve) shows the $\mathbf{k}_{2}^{2}$ distributions of the two - jet events. Compared to the full set of events (Fig. 12 dotted curve) one notices a slightly steeper decrease.

For the three jet events $(T<0.9)$ the opening angle $\Delta$ ranges between 1.2 and 2.2 (Fig. 13), i.e. we have a star - like configuration. Again, the gluon prefers to be in the forward direction (Fig. 12, dashed curve). However, compared to the two - jet events, the fall - off at large $\mathbf{k}_{2}^{2}$ is less steep. In other words, events with a large gluon transverse momentum are more likely to be three - jet like 

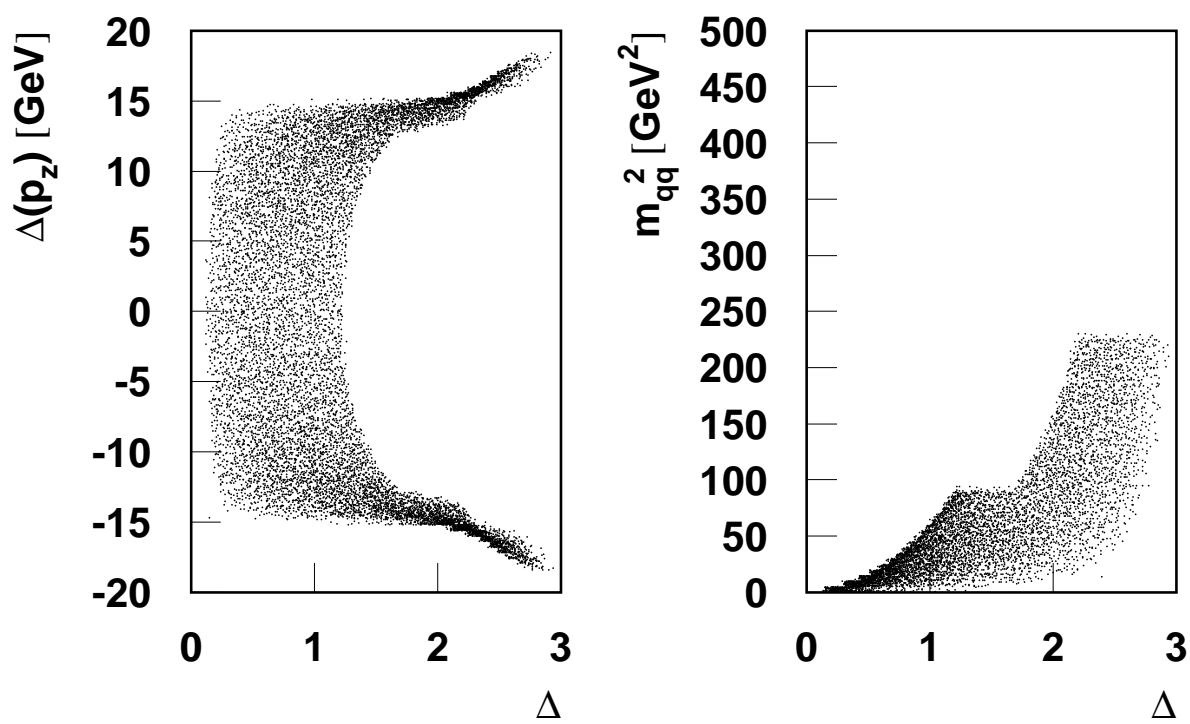

Figure 9: The difference of the longitudinal momenta of the two quarks along the $\mathrm{z}$ direction $\left(\Delta\left(p_{z}\right)\right)$ as a function of the opening angle $\Delta$ of the $q \bar{q}$ pair for thrust $T>0.9$. The second plot shows the correlation of the $q \bar{q}$-invariant mass and $\Delta$.

than those with a small transverse momentum. A few numbers: for $\mathbf{k}_{2}^{2}<10 \mathrm{GeV}^{2}$ the ratio of 3 jet to 2 - jet events is about 0.2 , whereas for $\mathbf{k}_{2}^{2}>10 \mathrm{GeV}^{2}$ this ration changes to 0.47 .

\subsection{HERA Region}

After this more general study we now turn to the HERA region. The results of the previous study should apply as long as the diffractive mass is sufficiently large: only under these conditions we have a sufficiently large range in transverse momenta. In HERA kinematics this requirement forces us into the (difficult) region of very small $\beta$ : for $Q^{2}=5 \mathrm{GeV}^{2}$ and $M^{2}=500 \mathrm{GeV}^{2}$, we would need $\beta=0.01$. In a first step we have repeated our above analysis for this region, and we have found that, indeed, the previous conclusions on our model still apply. In this region, however, we expect secondary exchanges to become important, and the theoretical interpretation of a jet analysis becomes more complicated.

A more realistic $\beta$-region is $\beta=0.1$. In the HERA region of not too large $Q^{2}$ we are then limited in $M^{2}$. This restriction, together with the lower cutoffs on the transverse momenta and the virtualities of the partons, lead to a severe limitation of the phase space for the transverse momenta of the jets. In our numerical analysis we have chosen $Q^{2}=5 \mathrm{GeV}^{2}$ and $M^{2}=45 \mathrm{GeV}^{2}$. We do not impose a cut on $m_{q \bar{q}}^{2}$ or $\alpha_{1}$ and $\alpha_{2}$. Such a cut would suppress the cross section by a factor of around 10 rendering the analysis meaningless. Our strategy will be a comparison with the asymptotic situation. From this we can deduce how far we can trust our asymptotic formulae for the HERA kinematics.

We again begin with the $k_{t}$-spectra (Fig. 14) for the proposed HERA kinematics, and we fit the power $p$. For the quark-spectrum we find $p=1.8$ and for the gluon-spectrum $p=2.3$. Both 

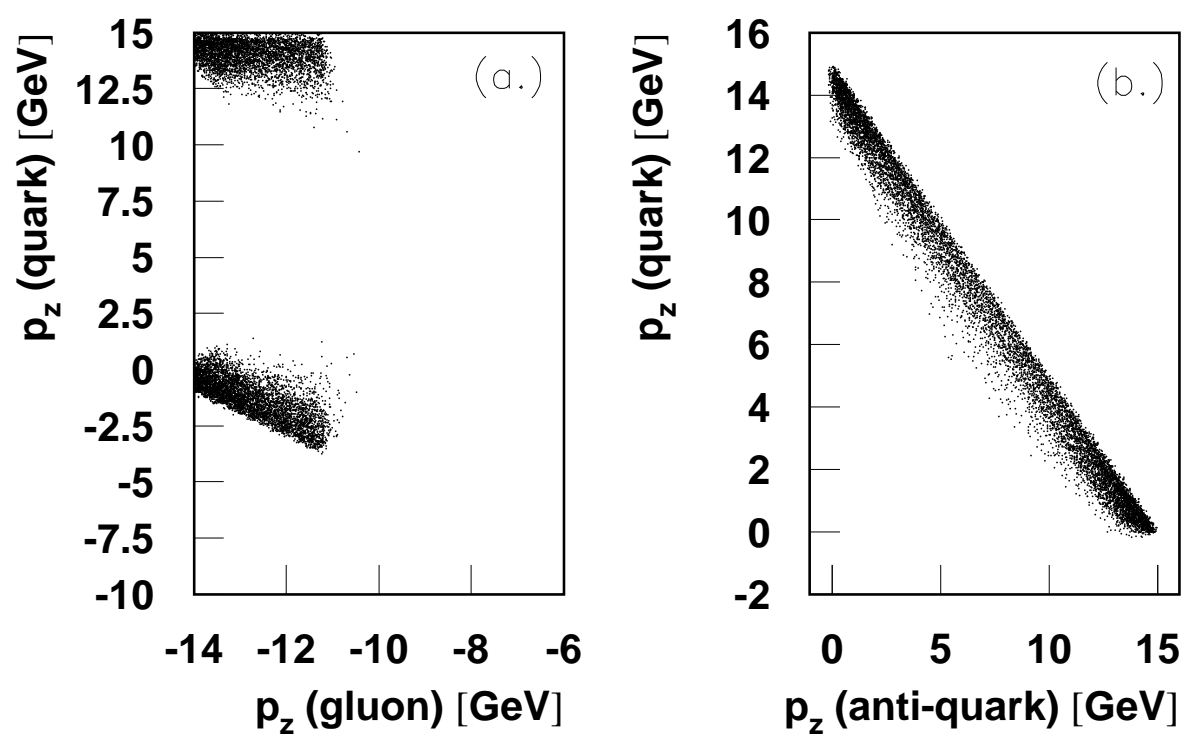

Figure 10: $a$. The correlation of the longitudinal quark and gluon momentum for $T>0.9$ and $\Delta>\pi / 2$ (large opening angle). $b$. The longitudinal momenta of the quark and antiquark for $T>0.9$ and $\Delta<\pi / 2$ (small opening angle). Both momenta are strongly correlated.

distributions have become steeper as compared to the asymptotic case which is mainly due to the stronger restriction in the phase space. The available energy is approximately a factor 10 smaller now. We also learn that a measurement of the $k_{t}$-spectrum cannot directly be related to the analytic estimates we have done in the previous section. In order to test to what extent the usual assumption of strong ordering between the transverse momenta of quarks and gluons is satisfied we compute the fraction of the cross section where $\mathbf{k}_{2}^{2}<\min (|\hat{t}|,|\hat{u}|)$ : we find that approximately only $2 / 3$ of the cross section satisfies this constraint, whereas $1 / 3$ lies outside this region. Next we have a look at the thrust distribution (Fig. 15). It is much broader than in the asymptotic case. Since $M^{2}$ is no longer that large, the relative proportion of transverse to longitudinal momenta is much higher than before, and therefore leads to an increase of three-jet events (around $60 \%$ of the events have a thrust smaller than 0.9). If we ask for two-jet events, i.e. thrust $T>0.9$, then we find again two configurations (Fig. 16), one with rather small opening angle between the quarks and a second with large opening angle. The distribution of $\Delta p_{z}$ versus opening angle $\Delta$ looks similar to the corresponding plot for asymptotic energies which makes us believe that the asymptotic formulae also work properly for HERA kinematics.

Table 1 shows the integrated $q \bar{q} g$ diffractive jet cross sections. For comparison, we also present dijet cross sections from $q \bar{q}$ production. We have integrated over the kinematic region $10 \mathrm{GeV}^{2}$ $<Q^{2}, 50<W<220 \mathrm{GeV}, x_{\mathbb{P}}<10^{-2}$, where we have integrated over $t$, assuming an exponential $t$ dependence. In the first row we present cross sections for the case where all parton $k_{t}^{2}$ are larger than $2 \mathrm{GeV}^{2}$ in addition to $|\hat{t}|,|\hat{u}|>2 \mathrm{GeV}^{2}$, and in the second row the corresponding cut is $5 \mathrm{GeV}^{2}$. For comparison, in the same kinematic region the inclusive diffractive cross section (for three flavors) is estimated to be $\sim 2.5 \mathrm{nb}$, i.e. for the lower $k_{t}$ cut the combined $q \bar{q}$ and $q \bar{q} g$ jet rate 


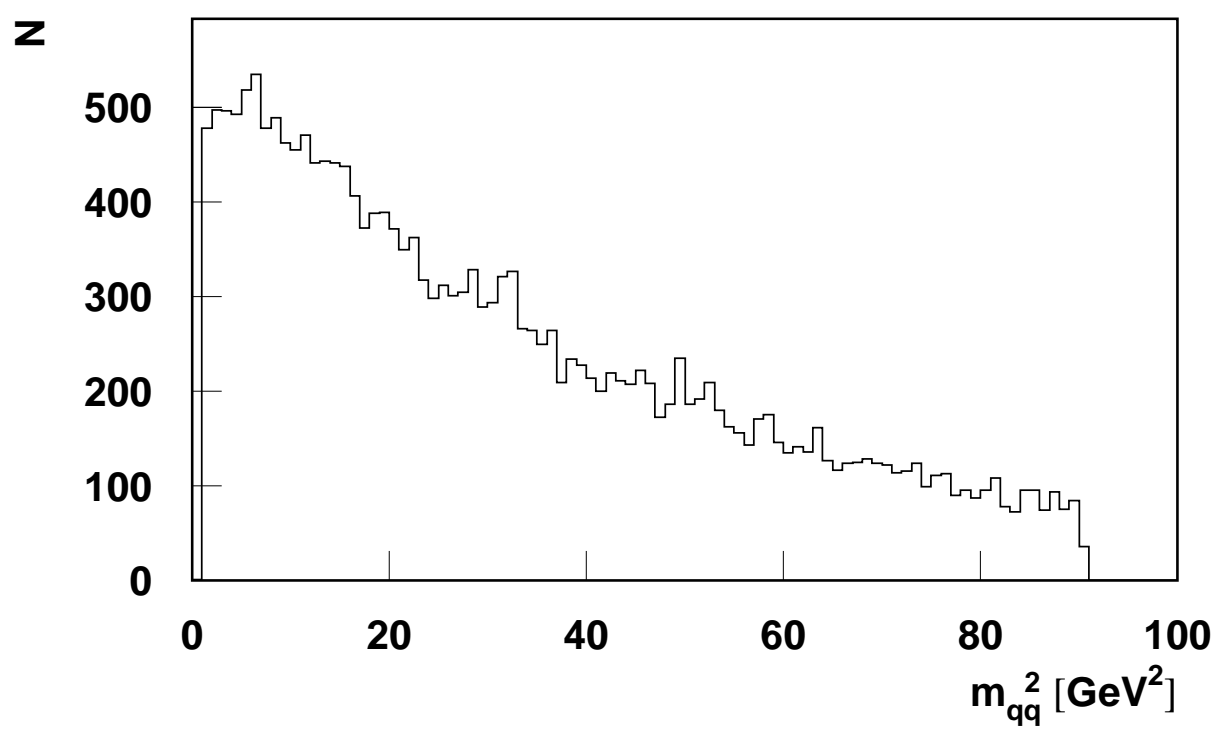

Figure 11: The distribution of the mass $m_{q \bar{q}}^{2}$ of the $q \bar{q}$ pair for thrust $T>0.9$

amounts to approximately $8 \%$ of the diffractive cross section. A look at the $k_{t}$ spectra indicates that the $q \bar{q} g$ jet rate strongly increases if we lower the cutoffs (entering a region where our formula

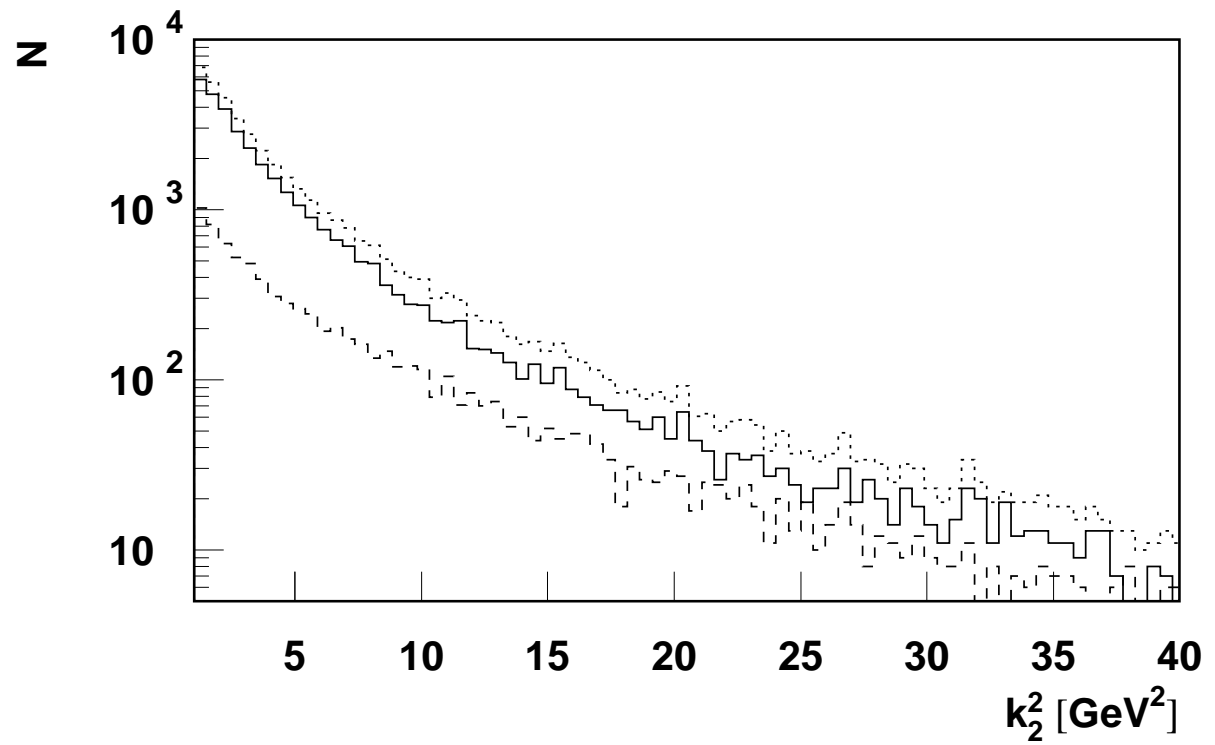

Figure 12: The transverse momenta of the gluon for events with $T>0.9$ (solid line), for $T<0.9$ (dashed line) and for all events (dotted line). 


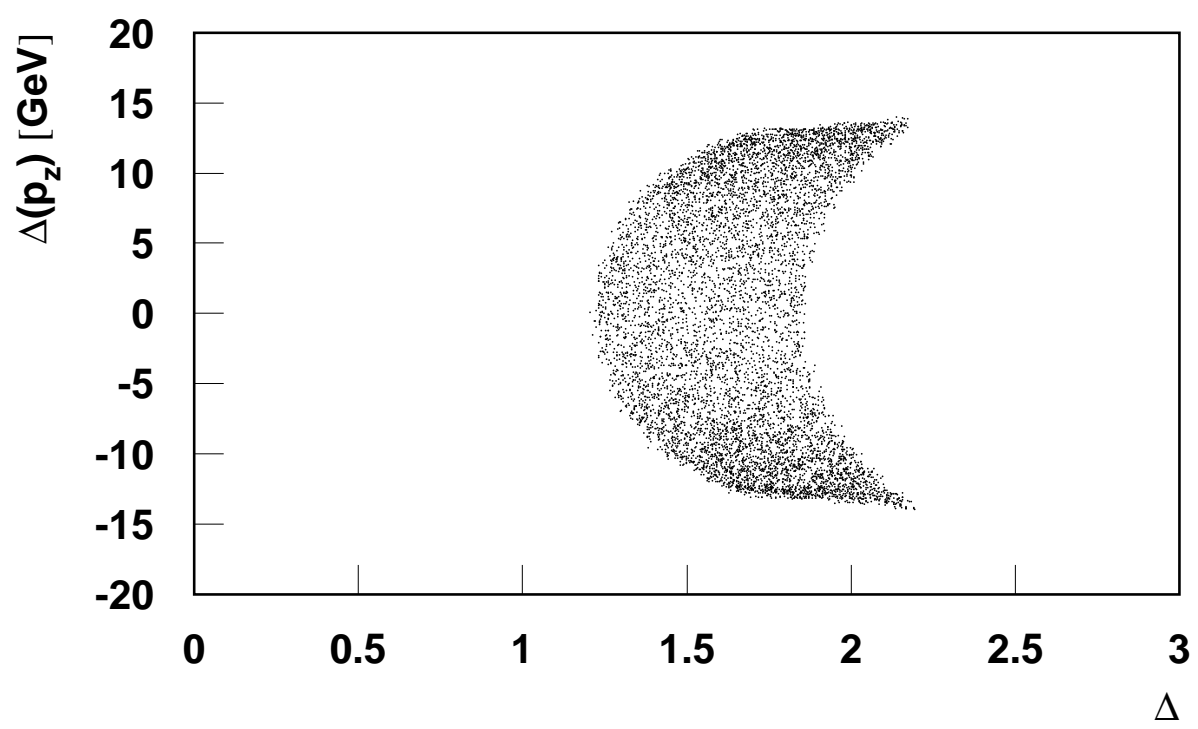

Figure 13: The difference of the momenta of the two quarks along the $\mathrm{z}$ direction $\left(\Delta\left(p_{z}\right)\right)$ as a function of the opening angle $\Delta$ of the $q \bar{q}$ pair for thrust $T<0.9$.

requires a modification of the gluon structure function): this indicates that the largest part of the $q \bar{q} g$ cross section might come from an intermediate region where, in particular, the gluon $k_{t}$ is larger
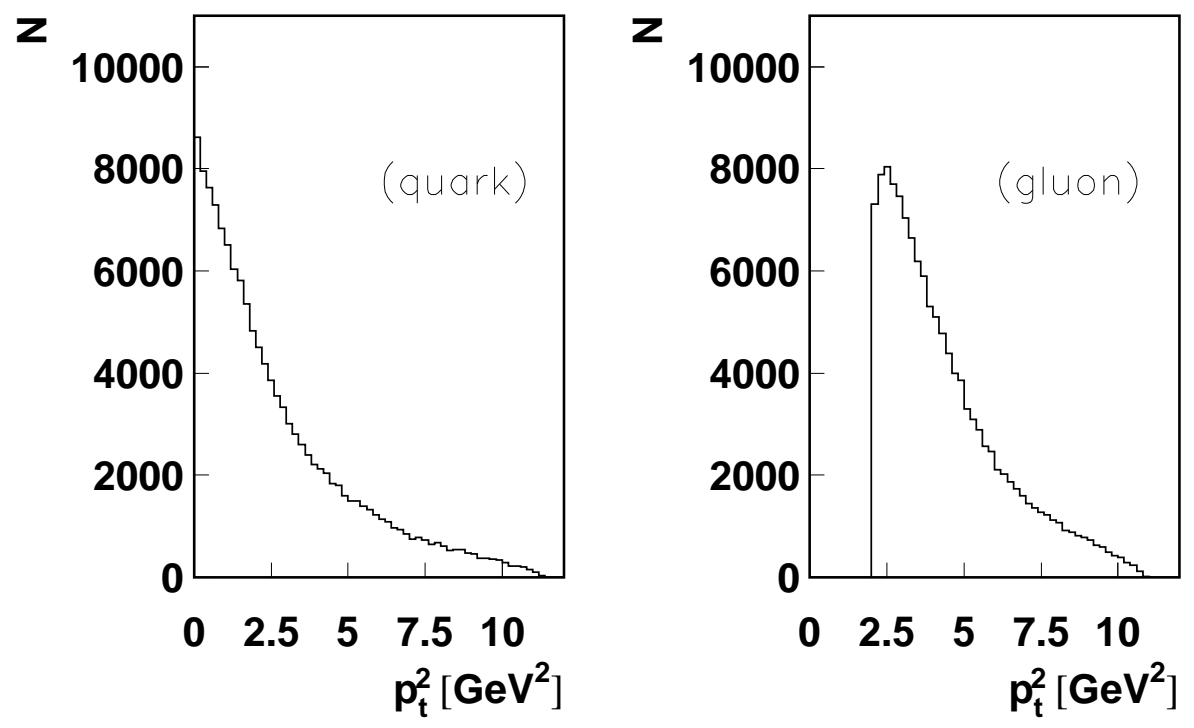

Figure 14: The distribution of transverse momenta for quarks and gluons. 


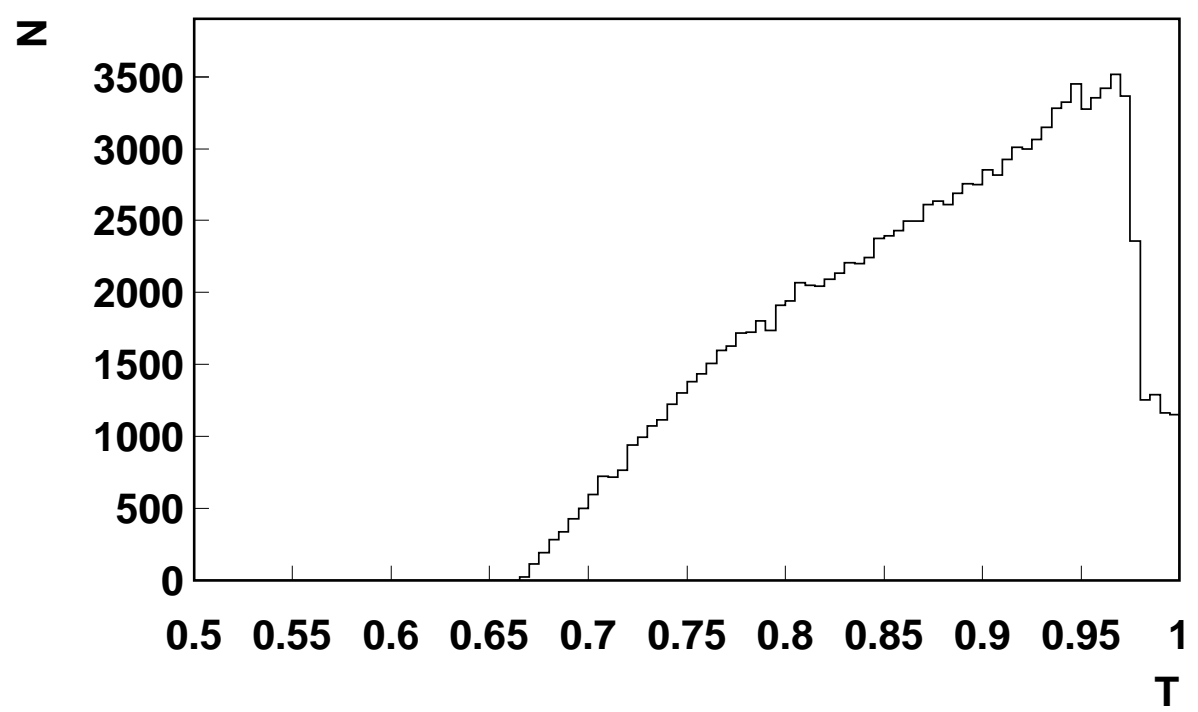

Figure 15: The distribution of thrust $T$ at HERA energies.

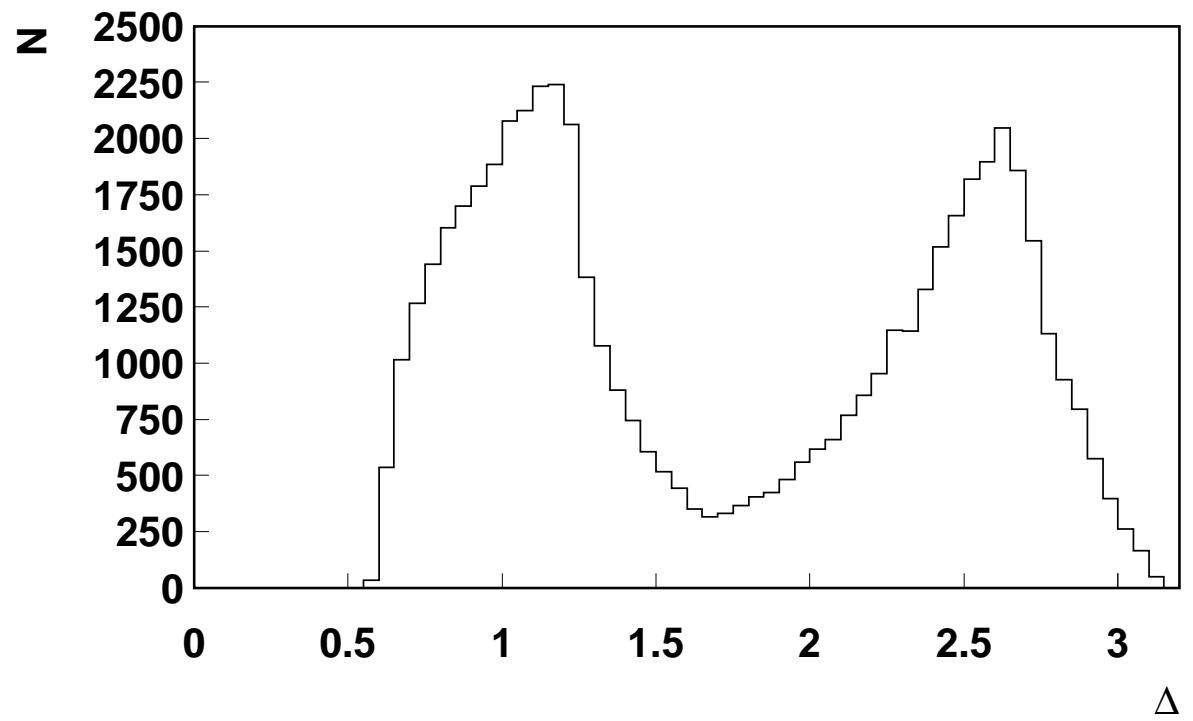

Figure 16: The opening angle $\Delta$ for $T>0.9$ at HERA energies.

than the soft Pomeron scale but lower than the cutoff values used in our analysis.

As in the case of diffractive production of two jets we expect the $q \bar{q} g$ jet cross section to rise in $1 / x_{\mathbb{P}}$; for the special case $\mathbf{k}_{2}^{2} \ll \mathbf{k}_{1}^{2}$ such a behavior follows immediately from eq. (4.5): $\sigma \sim\left[x_{\mathbb{P}} g\left(x_{\mathbb{P}}, \mu^{2}\right)\right]^{2}$. In Fig. 17 we show the cross section as a function of $x_{\mathbb{P}}$ at fixed $\beta$. In the 


\begin{tabular}{|c|c|c|}
\hline kinematic cut & dijet & $q \bar{q} g$ \\
\hline \hline $2 \mathrm{GeV}^{2}$ & $117 \mathrm{pb}$ & $66 \mathrm{pb}$ \\
$5 \mathrm{GeV}^{2}$ & $55 \mathrm{pb}$ & $9.2 \mathrm{pb}$ \\
\hline
\end{tabular}

Table 1: Integrated cross sections for diffractive $q \bar{q}$ and $q \bar{q} g$ production in the kinematic region defined by: $10 \mathrm{GeV}^{2}<Q^{2}, 50<W<220 \mathrm{GeV}, x_{\mathbb{P}}<10^{-2}$. The $t$ dependence is assumed to be exponential and the $t$ integral is performed. The kinematic cut requires all parton $k_{t}^{2}$ and in the case of $q \bar{q} g$ also $|\hat{t}|,|\hat{u}|$ to be greater than the value specified.

\section{$\times 10^{-2}$}

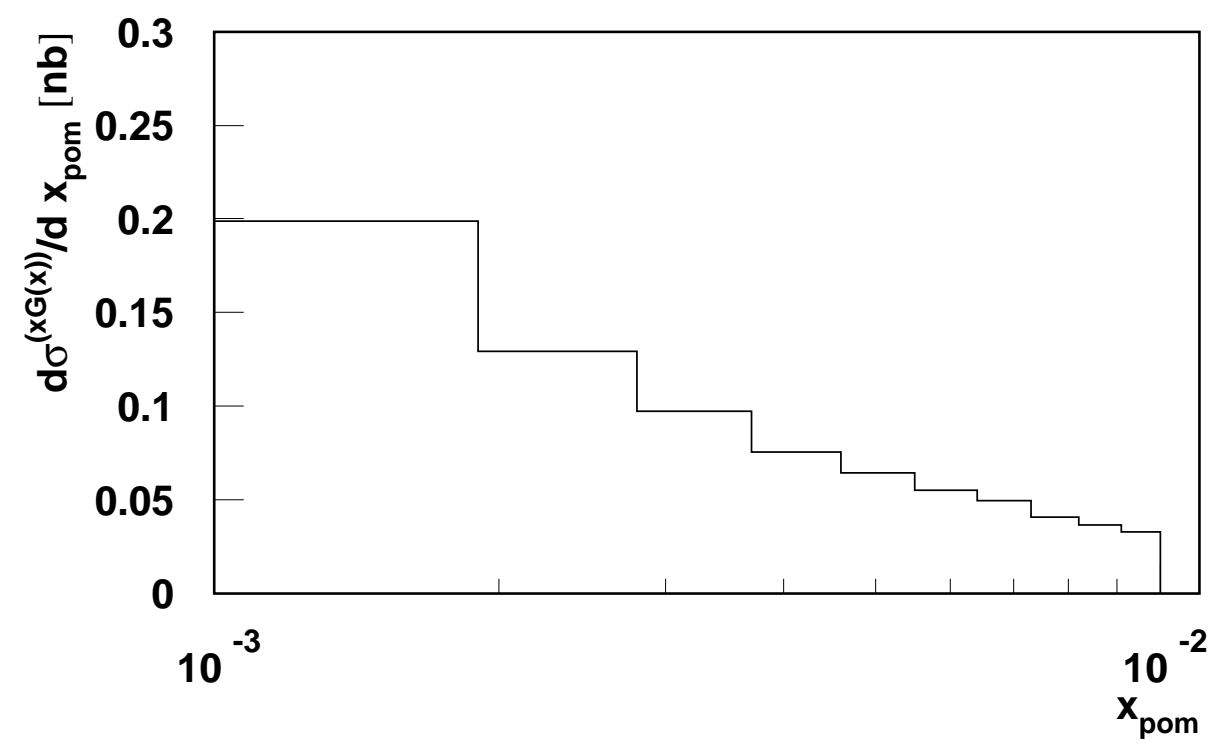

Figure 17: The cross section as a function of $x_{\mathbb{P}}$ at fixed $\beta=0.1$. The cross section is integrated over $t$.

region $\beta=0.1$ (i.e. large diffractive mass) we expect our formula to be applicable. One can see the steep rise of the cross section at small $x_{\mathbb{P}}$, a similar behavior as observed already in the dijet case. This rise is in qualitative agreement with the approximation eq. (4.5). As we have indicated in the introduction, we expect the $q \bar{q} g$ diffractive final state to dominate in the region of large diffractive masses (small $\beta$ ). In Fig. 18 we show, for different $\beta$ bins, the integrated cross sections of the two diffractive processes $q \bar{q}$ and $q \bar{q} g$ production. One sees that the $q \bar{q} g$ becomes dominant approximately for $\beta<0.2$. It is interesting to note that this $\beta$-value is consistent with the fit to the diffractive cross section in [3]: this fit also suggests that the $q \bar{q} g$ contribution begins to dominate for $\beta \approx 0.2$. In [4], the solution with a large $\gamma$-value is closer to our estimate than the small- $\gamma$ solution.

Next we study in more detail the specific properties of the $q \bar{q} g$ system, in particular the angular distribution of the three jets. We begin with a scatter plot of the angles of the quarks, keeping the angle of the gluon fixed. Quarks angles are defined as angles between quark and photon directions. 


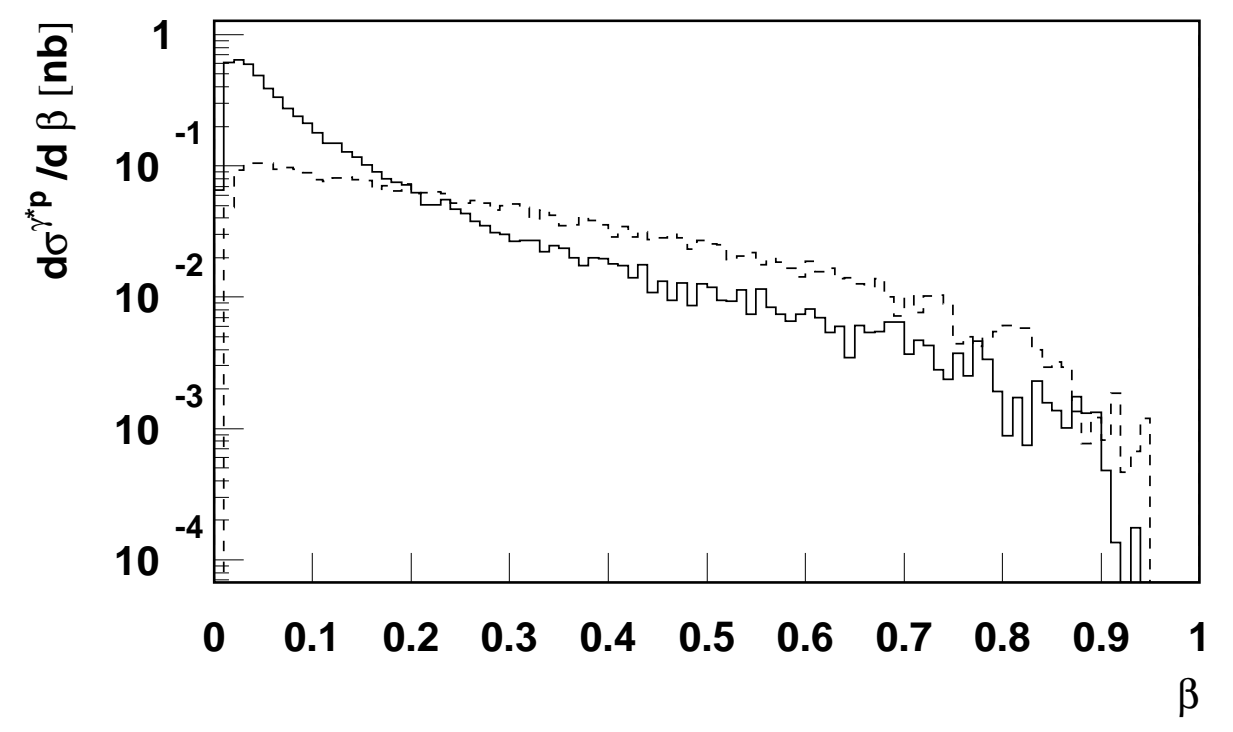

Figure 18: Diffractive jet cross sections $d \sigma / d \beta$ at $t=0$ for $0.01<y<0.9, Q^{2}>5$, and $x_{\mathbb{P}}<0.01$. All $k_{T}^{2}$ 's are restricted to be larger than $2 \mathrm{GeV}^{2}$. The solid lines shows the $q \bar{q} g$ final state, the dotted one the final state $q \bar{q}$.

In Fig. 19 we show the results for three different regions of the angle $\theta_{2}$ of the gluon. According to eq. (2.22), this angle is related to the transverse momentum $\mathbf{k}_{2}$ of the gluon. At small gluon angle (direction of the gluon close to the proton (Pomeron) direction, Fig. 19a.), in most of the events both quark and antiquark are moving not far from the photon direction (i.e. both their $\cos \theta$ 's are close to 1 ). However, there are also events in which one of the quarks stays close to the photon direction, whereas the other one sticks out at a larger angle. At large angle of the gluon (gluon direction orthogonal to the photon-Pomeron beam axis, Fig. 19c.), on the other hand, the quarks tend to move in opposite directions, one in the photon direction and the other in the proton direction. Correspondingly, at small gluon angle the opening angle $\Delta$ between the quarks prefers to be around $\pi / 3$ (Fig. 20a.), whereas for large gluon angle (Fig. 20c.) $\Delta$ peaks at a value slightly above $2 \pi / 3$. In all these discussions it should be made clear, that case (c.) lies at the edge of the region of validity of our approximation. A reliable calculation of this kinematic region requires a complete NLO calculation of diffractive $q \bar{q}$ and $q \bar{q} g$ production, in particular virtual corrections to $q \bar{q}$ production and real gluon emission from quark lines.

From these considerations we arrive at the following picture:

- At small transverse momenta of the gluon, we have mainly two-jet configurations where the quarks travel in approximately the same direction with an opening around $\pi / 3$, carrying similar longitudinal momenta. But the two quarks can also appear in a more asymmetric configuration, where one of the quarks has a much larger longitudinal momentum compared to the other quark, and moves in approximately the direction opposite to the gluon. The quark with the smaller longitudinal momentum then sticks out from the $z$-axis.

- In the other extreme, where the gluon is emitted perpendicular to the $z$-axis, we observe a 


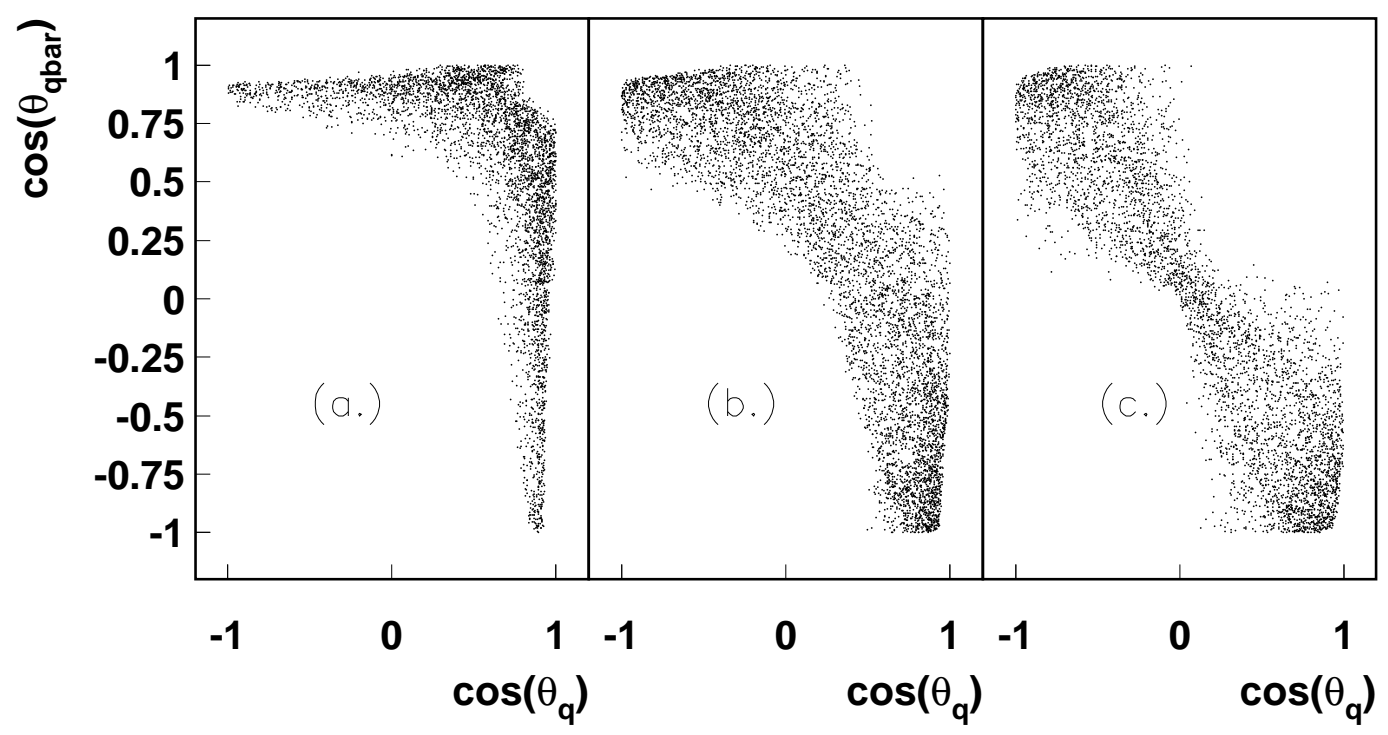

Figure 19: The $\theta$ angle of the quark versus the $\theta$ angle of the antiquark. (a.) for $-1<$ $\cos \left(\theta_{\text {gluon }}\right)<-0.8,(b$. $)$ for $-0.6<\cos \left(\theta_{\text {gluon }}\right)<-0.4$, (c.) for $-0.2<\cos \left(\theta_{\text {gluon }}\right)<0.0$, at $\beta=0.1$ and $Q^{2}=50 \mathrm{GeV}^{2}\left(M_{X}^{2}=45 \mathrm{GeV}^{2}\right)$.

'Mercedes - star" like configuration, in which both quarks carry longitudinal momenta of similar value, but in opposite direction, and the cosine of the opening angle lies between two and three.

- At medium transverse momentum $\mathbf{k}_{2}$ of the gluon, all configurations discussed above, are possible.

\section{Conclusions}

In this paper we have presented an analysis of hard diffractive $q \bar{q} g$ jets with large transverse momenta. Our study is motivated by the observation that in DIS diffractive dissociation, the Pomeron intercept lies above the value seen in purely hadronic interactions: this suggests that the diffractive final state contains a rather large 'hard' component. A natural candidate for hard final states are jets. The investigation of diffractive $q \bar{q} g$ production presents a generalization of diffractive dijet production [6]. Our calculation is restricted to the low- $\beta$ region (large diffractive masses), and we have been working in the leading-log $1 / \beta$ approximation. At the same time, the transverse momenta of the outgoing partons are not restricted by a strong ordering requirement. In the low- $\beta$ region the emitted gluon carries a small momentum fraction of the incoming photon, i.e. in rapidity it is closer to the proton than the two quarks (Fig. 32).

The analytic expression for the cross section formula in impact parameter space is remarkably simple. It illustrates that the concept of a photon wave function holds even beyond the approximation, where the transverse momentum of the emitted gluon is much softer than the quarks. For 


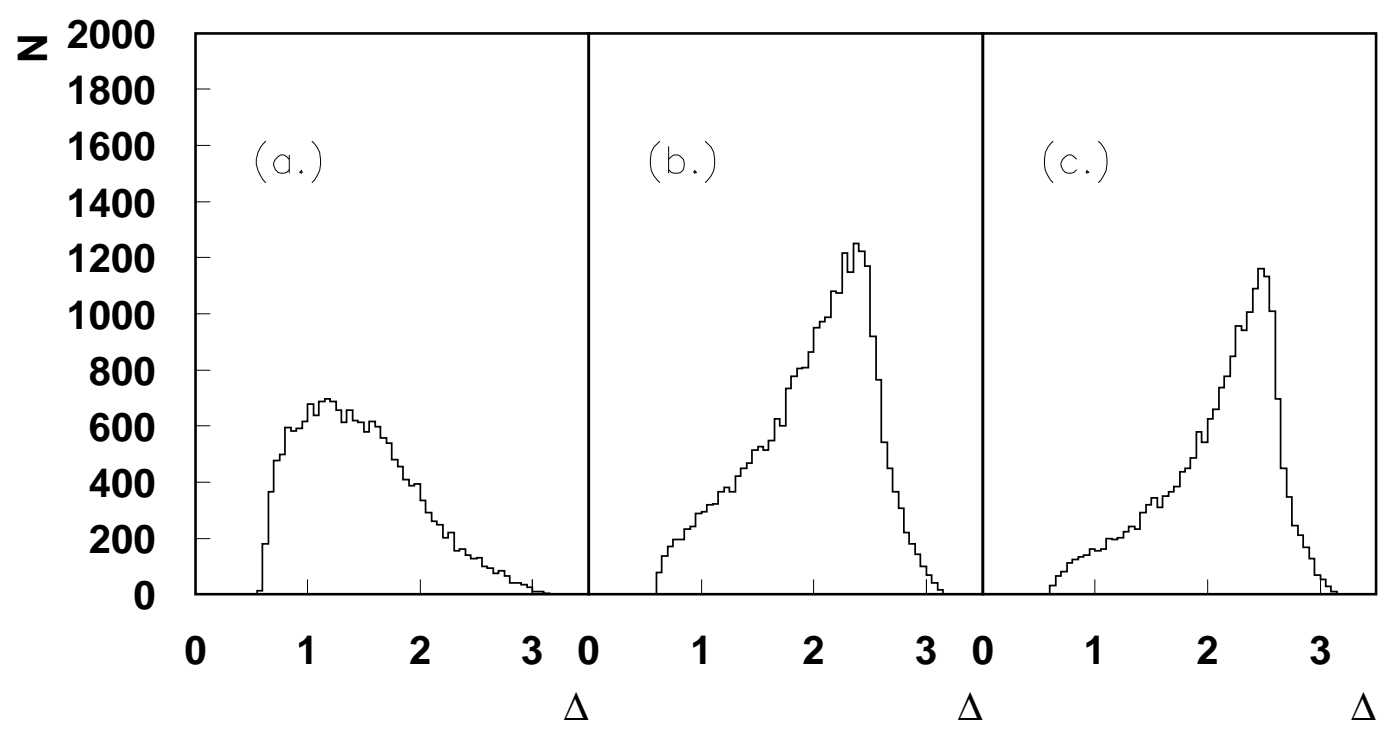

Figure 20: The opening angle $\Delta$ between the quarks. (a.) for $-1<\cos \left(\theta_{\text {gluon }}\right)<-0.8$, (b.) for $-0.6<\cos \left(\theta_{\text {gluon }}\right)<-0.4$, (c.) for $-0.2<\cos \left(\theta_{\text {gluon }}\right)<0.0$, at $\beta=0.1$ and $Q^{2}=50 \mathrm{GeV}^{2}\left(M_{X}^{2}=45 \mathrm{GeV}^{2}\right)$.

special kinematic limits we have obtained even simpler expression for the cross section formulae.

In a first exploratory numerical study we have calculated jet cross sections. Depending upon the lower limit of the transverse momenta, the combined $q \bar{q}$ and $q \bar{q} g$ jet cross section can be as large as $8 \%$ of the inclusive diffractive cross section. We have also looked into the spatial distribution of the three-parton final state. If (in the $\gamma^{*}-\mathbb{P}$ CM-system) the gluon jet is close to the Pomeron direction, the quark - antiquark pair mainly forms a single (although somewhat broader) jet opposite to the gluon jet. In the extreme case where the emitted gluon is perpendicular to the photon - Pomeron axis, a clean three-jet configuration emerges. However, in this region the cross section will be small. For a diffractive jet analysis, it is important to keep in mind that quite a large fraction of the $q \bar{q} g$ final states will appear as a two-jet configuration, and it will be difficult to separate them from the $q \bar{q}$ dijet final states.

Experimentally the analysis of two or three jet events is not easy. A more promising way may be a thrust or $E_{T}$ analysis. To this end, it would be very desirable to have a complete NLO calculation of diffractive final states with two or three jets.

\section{Acknowledgement}

Part of this work was done while one of us (J.B.) was visiting the Fermilab Theory Division. He gratefully acknowledges their hospitality. 


\section{References}

[1] H1 Collaboration, Z. Phys. C 76 (1997) 613.

[2] ZEUS Collaboration, Eur. Phys. J. C 6 (1999) 43.

[3] J. Bartels, J. Ellis, H. Kowalski, and M. Wüsthoff, hep-ph/9803497, to be published in Eur. Phys. J. C.

[4] J. Bartels, C. Royon, hep-ph/9809344 and DESY 98-132..

[5] S.J. Brodsky, L. Frankfurt, J.F. Gunion, A.H. Mueller, M. Strikman, Phys. Rev. D 50 (1994) 3134; L. Frankfurt, W. Koepf, M. Strikman, Phys. Rev.D 54 (1996) 3194; J.C. Collins, L. Frankfurt, M. Strikman, Phys. Rev. D 56 (1997) 2982.

[6] J. Bartels, H. Lotter, M. Wüsthoff, Phys. Lett. B 379 (1996) 239; ERRATUM-ibid. B 382 (1996) 449.

[7] N. Nikolaev, B.G. Zakharov, Z. Phys. C 53 (1992) 331.

[8] E. Gotsman, E. Levin, U. Maor, Nucl.Phys. B 493 (1997) 354; Nucl. Phys. B 493 (1997) 354.

[9] J. Bartels, C. Ewerz, H. Lotter, M. Wüsthoff Phys. Lett. B386 (1996) 389 and references therein

[10] H1 Collaboration, Eur. Phys. J.C 6 (1999) 421.

[11] M.G. Ryskin, Sov. J. Nucl. Phys. 52 (1990) 529.

[12] M. Wüsthoff, Phys. Rev. D 56 91997) 4311, K. Golec-Biernat, M. Wüsthoff, in preparation.

[13] K. Golec-Biernat, M. Wüsthoff, Phys. Rev. D 59 (1999) 014017.

[14] L. Frankfurt, M. Strikman Phys. Rev. Lett. 63 (1988) 1914.

[15] J.C. Collins, Phys. Rev. D 57 (1998) 3051.

[16] J. Bartels, Nucl. Phys. B 175 (1980) 365.

[17] J. Bartels, M. Wüsthoff, Z. Phys. C 66 (1995) 157

[18] N. Nikolaev, B.G. Zakharov, Z.Phys. C 64 (1994) 631.

[19] A.H. Mueller, B. Patel, Nucl. Phys. B 425 (1994) 471.

[20] M.G. Ryskin, proceedings of the workshop 'Physics at HERA', Hamburg, Oct. 29-30,1991.

[21] E. Levin, M. Wüsthoff, Phys.Rev. D 50 (1994) 4306.

[22] H. Jung, Comp. Phys. Comm. 86 (1995) 147.

H. Jung, The RAPGAP Monte Carlo for Deep Inelastic Scattering, version 2.07, Lund University, 1999, (to be published)

http://www-h1.desy.de/ jung/rapgap.htm.

[23] M. Glück, E. Reya, A. Vogt, Z. Phys. C 67 (1995) 433. 